\title{
FD MAC Protocol Design for Co-Existing WLANs in 5G Cellular Networks
}

\author{
S. Diakite ${ }^{1}$ (D), F. M. Rizo ${ }^{2}$ (D), S. Y. M. Bandiri ${ }^{3}$ (D) \\ ${ }^{1}$ Institut des Sciences Appliquées (ISA), Département de Génie Electrique et Informatique Industrielle \\ (GEII), Street 405, 359, Bamako, Mali.souleymane.diakite@isamali.org, \\ ${ }^{2}$ Universidad Tecnológica de La Habana (CUJAE), Departamento de Communicaciones Inalámbricas, \\ Street 114, 11901, Havana, Cuba.marante@tele.cujae.edu.cu \\ ${ }^{3}$ Universidade da Integração Internacional da Lusofonia Afro-Brasileira (UNILAB), Departamento da \\ Telecomunicacion, Street 11,03, Ceará,Brazil.bandiri@unilab.edu.br
}

\begin{abstract}
In this paper, we design full-duplex (FD) medium access control (MAC) protocol for co-existing wireless local area networks (WLANs) in 5G cellular networks (WCFD-MAC). Our design considers some significant features of $5 \mathrm{G}$ networks, exceptionally, beamforming and FD capabilities at both, the base station and user equipment. FD communications may generate interferences, namely self-interference (SI) in bidirectional FD (BFD) and interuser interferences in three-node FD (TNFD). Several efforts have been performed to mitigate the SI in BFD communications. However, the inter-user interferences in TNFD are still considered as a major drawback in FD networks. These interferences must be mitigated by an efficient MAC protocol design. WCFD-MAC protocol allows two neighboring users to simultaneously participate in TNFD communication without interfering with each other by using directional transmission and a three-node angle condition (TAC). Directional transmission combined with TAC increases system throughput. WCFD-MAC protocol allows a new half-duplex (HD) communication scheme referred to as three-node HD (TNHD). This scheme may occur when bidirectional FD (BFD) and TNFD communications cannot occur. TNHD scheme includes device-todevice (D2D) communication and allows energy efficiency, which is one of the keys requirements of $5 G$ wireless networks. Simulations results show that WCFD-MAC protocol achieves higher throughput than existing works in the literature.
\end{abstract}

Index Terms - Directional transmission, Inter-user interference, Medium Access Control (MAC), Three-node FD communication.

\section{INTRODUCTION}

Multi-radio access technology (RAT) carrier aggregation is a technique that allows channels from different RATs to be aggregated and allocated to the end-user [1]. LTE-WLAN aggregation (LWA) emerged as a new alternative technology to LTE-U/LAA (LTE-Unlicensed/LTE Licensed Assisted Access). Release 13 includes LTE-WLAN aggregation where LTE (Long Terme Evolution) and WLAN (Wireless Local Area Network) are aggregated at the packet data convergence protocol (PDCP) layer, using a framework very similar to dual connectivity [2]. Furthermore, release 13 specifications allow aggregation of a primary cell, operating in licensed spectrum to deliver critical information and guaranteed quality of service (QoS), and a secondary cell, operating in an unlicensed 
spectrum to opportunistically boost data rate. The increasing demands for high data traffic stimulated the development of 5G mobile networks [3]. FD (Full-Duplex) technology is one of the keys techniques that will support the immense traffic demands in $5 \mathrm{G}$ networks by increasing spectral efficiency without requiring any additional frequency resources. 5G Wireless networks will also use higher frequency bands (mmWave: millimeter wave) and massive MIMO (Multiple-Input MultipleOutput) technology [4] that will allow beamforming/directional antenna transmission [5]. The characteristics of the future co-existing WLANs in $5 \mathrm{G}$ cellular networks have to satisfy most of the defined requirements for $5 \mathrm{G}$ cellular networks, such as FD capability, massive MIMO and mmWave technologies, beamforming/directional antennas capability, low latency, high throughput, energy and spectral efficiencies. All or most of these requirements need to be taken into consideration when designing an efficient MAC protocol [6], for 5G cellular co-existing WLANs. Since there is a difference in the characteristics of mmWave to the conventional signals, PHY and MAC (Medium Access Control) layer aspects need to be changed [7]. Moreover, the IEEE 802.11 standard MAC protocol has been designed for omnidirectional antennas and HD (Half-Duplex) communications. The use of directional antennas can be beneficial for wireless networks, since it can significantly increase spatial reuse and reduce network latency [8]. Thus, the network throughput is improved [9].

There are two scenarios in the existing FD MAC protocol: bidirectional FD (BFD) and three-node FD (TNFD) [10], [11]. In a covered small cell area, some interferences may arise from two simultaneously transmissions due to the use of one frequency band for all the communications. For the BFD case, several efforts have been realized to mitigate the self-interference (SI) at each transceiver [12], [13]. The inter-user interference in TNFD communication is still considered as one of the main drawbacks in FD networks [14]. The authors in [10] proposed an FD MAC protocol-based infrastructure design for heterogeneous wireless LANs (HFD-MAC). To address inter-user interference in TNFD communications, the proposed design only avoids TNFD communication when the secondary transmitter (ST) and the primary receiver (PR) are in the transmission range of each other as shown in Fig. 1 a). A similar condition is also applied to avoid inter-user interference between the primary transmitter (PT) and secondary receiver (SR) as depicted in Fig. 1 b). However, in our MAC protocol design, two users can simultaneously participate in a TNFD communication if an angle condition is satisfied between them, such that the inter-user interference is mitigated even if they are in the transmission range of each other. Note that in this scenario, TNFD communication may occur with high probability in our FD MAC protocol in comparison to HFD-MAC protocol. Moreover, in the existing FD MAC protocol, when the base station (BS) has to send a data packet to a user equipment (UE) located at the cell boundary, which has no data packet to send to the BS, and the BS has no data packet to send to another UE apart from the boundary UE, HD communication may occur. In this case, a new HD scheme is introduced in our FD MAC protocol to enhance the cell boundary UE throughput. This new HD scheme is referred to as three-node HD (TNHD) in this paper. 
After exchanging all the necessary control frames, the BS will transmit the data packet to the intermediate UE, which will forward it to the cell boundary UE. In the TNHD communication scheme, the data packet is forwarded from the BS to an intermediate UE and then to the cell boundary UE. Thus, TNHD takes into account D2D communication [7]-[15], which may enhance the energy consumption for both, the BS and the boundary cell UE, and this is one of the main requirements of the 5G cellular networks [16], [17].

The network where WLAN standards and cellular standards are integrated [18], [19], is referred to as WLAN-cellular integrated network. In this way, 5G cellular and WLAN systems are expected to coexist in order to support different use cases [2]. All the users in 5G networks should operate in multimode terminals that can function in both radio technologies: licensed/cellular and unlicensed/WLAN [1], [20]. Hence, this paper aims to design FD MAC protocol for the future co-existing WLANs in 5G cellular networks, referred to as WCFD-MAC (WLAN cellular FD-MAC). Our design addresses the inter-user interference in TNFD communication, and introduces a TNHD communications scheme. Furthermore, our design takes into account FD and beamforming/directional transmission capabilities at both, the BS and UE, and a deice-to device (D2D) communication scheme. The use of beamforming/directional transmission can increase network throughput by allowing spatial reuse. Other $5 \mathrm{G}$ networks requirements, such as network densification and efficient power allocation scheme are beyond the present work. However, enhancing the MAC protocol can significantly improve communication reliability and energy efficiency. Therefore, a suitable FD MAC protocol design is crucial to exploit all the above-mentioned advantages of FD technology while managing TNFD interferences in the context of $5 \mathrm{G}$ cellular networks. The main contributions of the paper are as follows:

- WCFD-MAC allows two neighbor UEs to simultaneously participate in TNFD communication without interfering with each other by performing an angle condition verification.

- A new HD scheme (TNHD) is proposed in our design, which can enhance the throughput of the UEs at the covered small cell border and contribute to the energy efficiency in $5 \mathrm{G}$ networks.

- Beamforming/directional antennas transmission is one of the keys requirements of $5 \mathrm{G}$ cellular networks and is considered in our MAC protocol design, unlike the existing FD MAC protocol in the literature.

- A network throughput analytical expression that incorporates BFD, TNFD, and HD communications schemes is derived based on Bianchi's model.

The remainder of this paper is organized as follows: Section II shows the related works and motivation, the network and antenna system models are highlighted in Section III, Section IV shows TNFD inter-user interferences and mitigation, Section V introduces the designed WCFD-MAC 
protocol, then the performance evaluation of our proposal is presented in Section VI, finally Section VII presents the conclusion and future works.

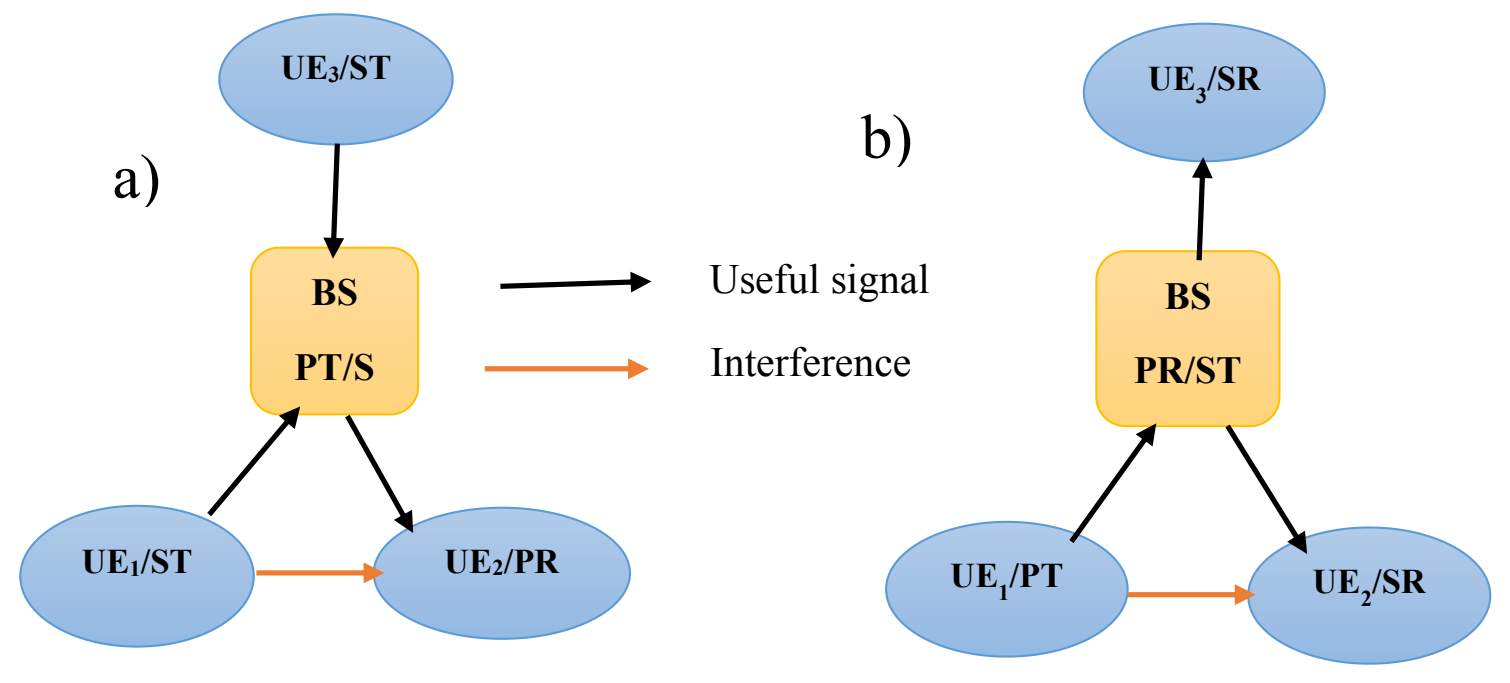

Fig. 1. Inter-user interference in TNFD: a) Interference from ST to PR; b) Interference from PT to SR.

\section{RELATED WORKS AND MOTIVATION}

The work presented in [1] designed a scalable MAC protocol for D2D communication for future $5 \mathrm{G}$ networks. In this design, a three tier $5 \mathrm{G}$ heterogeneous architecture was proposed, where a macro-cell BS co-exists with multiple small cell WLAN BSs, which allows traffic offload from the cellular system to the WLAN system in a dense environment. To address the resource allocation in the WLAN small cell, the authors designed a small cell MAC protocol (SC-MAC) based on the PCF (Point Coordination Function) access mechanism. The feasibility and practical deployment possibility of integrated LTE-WLAN systems for the 5G mobile networks have been highlighted in [21]. They stated that the small cell BS and the WLAN BS are wired, or collocated within an integrated physical element, and can exchange through an interface such as the X2 interface. The work in [22] considered mmWave integrated heterogeneous networks for $5 \mathrm{G}$ cellular, where both cellular and WLAN systems utilize mmWave for their communications. To handle the performance degradation challenges caused by the increasing density of the WLANs in $5 \mathrm{G}$ unlicensed network scenarios, a channel observationbased scaled backoff (COSB) mechanism is proposed in [23]. The proposed COSB is based on CSMA/CA without request to send clear to send (RTS/CTS) mechanism that can avoid the hidden terminal problem. Furthermore, COSB can reduce the collision probability in a dense deployment scenario, however, it will introduce more delay due to the observation time slots introduced after the channel become idle for DIFS time. Moreover, 5G networks FD capability and beamforming technology were not considered in the designed mechanism. In [24], FD MAC protocol design for 5G mobile wireless networks is provided. In the proposed MAC protocol, different schemes such as BFD, TNFD, and HD communications have been considered. The proposed design is based on the CSMA/CA and RTS/CTS mechanisms. However, beamforming/directional transmission and the 
inter-user interference in TNFD communication were not considered in the proposed design. In [25], another FD MAC protocol design for 5G cellular WLAN, called FDDS-MAC (FD dynamic scheduling MAC) aims to enhance the spectrum usage on FD communications in 5G mobile wireless networks, by increasing BFD transmission probability. FDDS-MAC uses pulse/tone signals instead of RTS/CTS for channel reservation, since those signals can be decoded in $5 \mu$ s. However, the decoding of the pulse/tone signal is challenging. Moreover, beamforming/directional transmission and TNFD scheme have not been considered in FDDS-MAC protocol design. To fully exploit FD benefit in heterogeneous wireless LANs, FD MAC (HFD-MAC) protocol based on CSMA/CA and RTS/CTS mechanisms have been proposed in [10], where the co-existence of the FD nodes and HD nodes is considered. HFD MAC protocol suppresses inter-user interference in TNFD communications, however, it does not allow two nodes to simultaneously communicate with the BS when they are in the transmission range of each other, in other when they are too closer.

Many existing FD MAC protocols have been addressed for ad-hoc mode networks deployment [26]-[30]. Although, some other works were focused on FD MAC protocol design for the infrastructure mode [31], [32]. However, there is still a lack of literature on FD MAC protocol-based infrastructure mode deployment. Moreover, from the mentioned works above, on the one hand, all of these works focused only on omnidirectional transmission, none of them addressed beamforming/directional transmission while designing FD MAC for WLAN based infrastructure mode. On the other hand, only HFD-MAC partially addressed the inter-user interference mitigation in TNFD communication for WLAN based infrastructure mode. Hence, in our FD MAC protocol design, we will take into account beamforming/directional transmission while addressing inter-user interference in TNFD communications, instead of mitigating the inter-user interference by avoiding TNFD communication when the corresponding users are in the transmission range of each other.

\section{NETWORK AND ANTENNA SYSTEM MODELS}

\section{A. $\quad$ Network model}

In this paper, we consider a covered small cell with WLAN BS. We assume that the BS and all the UEs use the same frequency band to exchange control frames and data packets. Each UE and BS have FD features, meanings that they can transmit and receive simultaneously using the same frequency band and can mitigate the self-interference. Also, both the BS and UEs can transmit directionally and omnidirectionally. To avoid deafness problems, in our FD MAC protocol all the control frames except for the ACKs frames are transmitted omnidirectionally, while the data packets are transmitted and received directionally by performing a directional beam in the direction of the receiver and transmitter, respectively. When the BS transmits a control frame omnidirectionally, all the UEs in the covered small cell can hear it and update their NAVs (Network Allocation Vectors) accordingly. Directional transmission will be possible after hearing a transmitted control frame and estimating the transmitter direction to then beam form in the direction of the incoming control frame 
signal. In the considered covered small cell three scenarios of communication, BFD, TNFD, and HD may occur. The communication can be initiated not only by the BS but also by a UE. BFD communication may occur when both transceivers have a data packet to each other. When one of them has no data packet for the other, TNFD communication may occur depending on if the BS finds another candidate to perform TNFD. Before performing TNFD, the BS will select the ST/SR such that inter-user interference is avoided by verifying an angle condition. If there is no candidate, HD communication will occur. To enhance the throughput of a UE located at the covered small cell border when HD communication becomes necessary, TNHD communication may be performed. To this end, the BS will transmit control frame containing its address and the addresses of both the UE located at the small cell border and the intermediate UE, which is located between the BS and the UE located at the covered small cell borders. After exchanging all the necessary control frames, the BS will transmit the data packet to the intermediate UE, which will forward it to the cell border UE. In this case, the BS is assumed to perform an efficient power control [9], [15], to avoid interference to the cell border UE. For the problem tractability, we consider the symmetric length of the uplink and downlink data traffic.

\section{B. Antenna system model}

The BS and all the UEs are assumed to be equipped with massive MIMO to perform beamforming technology using phased array systems (adaptive beamforming system) which can determine the angle of arrival (AoA) of the incoming signal and then switch the most appropriate beam. Also, this system can direct the beam in the exact direction needed and can move it in real time [33]. After hearing an RTD control frame signal from the transmitter, the indented receiver will beam form toward the transmitter direction, since it can estimate the exact location direction of the transmitter. Also, after hearing the corresponding CTSD, the transmitter can beam form toward the receiver direction. We assume that all the UEs and BS have the same beam width, $2 \beta$, which is symmetric concerning the desired transmission direction. We assume an adaptive beamforming system which can perform:

Omnidirectional transmission: omnidirectional transmission is used to transmit all the control frames apart from the ACK frame which is transmitted directionally. Thus, inter-user interference due to hidden/exposed can be avoided.

Directional transmission: only the data packets and ACK frames are transmitted directionally after the control frame exchanges. This will make possible TNFD communications in the covered small cell at the same time. Thereby, spatial reuse and spectral efficiency increase. Directional antennas transmission can allow a neighboring UE of the PT to simultaneously receive a data packet from the BS (PR and ST). Furthermore, it can allow a neighboring UE of the PR to simultaneously transmit a data packet to the BS (PT and SR) without interfering with the PR reception. Fig. 2 shows an example of a TNFD communication between the BS, $U E_{1}$ and $U E_{2}$, where $U E_{2}$ (PT) initiates the 
communication with the $\mathrm{BS}(\mathrm{PR} / \mathrm{ST})$ which has no data packet for $U E_{2}$, however, it has a data packet for to $U E_{1}(\mathrm{SR})$, then a TNFD communication can occur between the BS, $U E_{1}$ and $U E_{2}$, without interfering with each other even if $U E_{1}$ and $U E_{2}$ are in the transmission range of each other thanks to directional transmission.

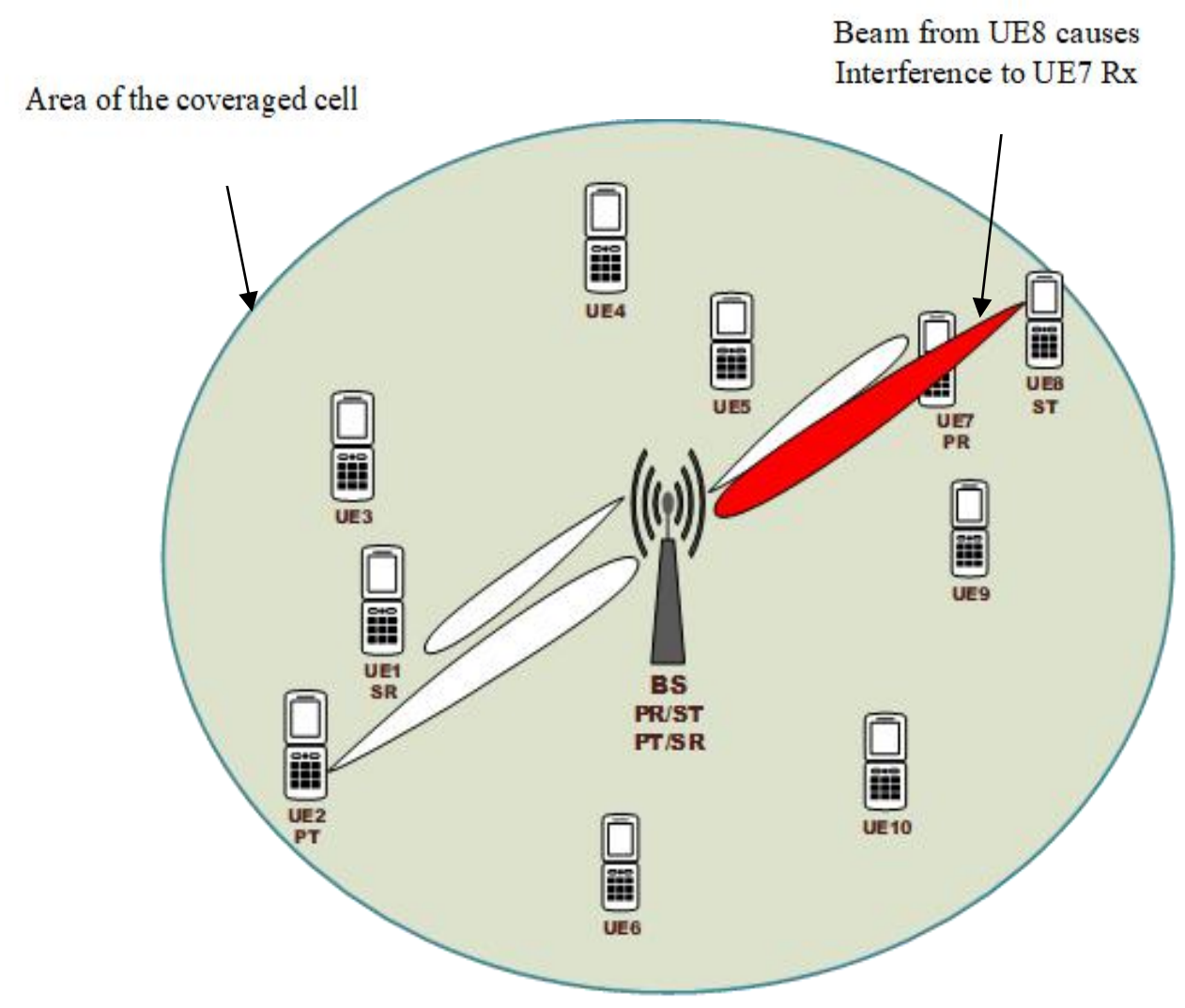

Fig. 2. Directional antennas transmission between BS and UEs in the covered cell.

In TNFD communication, interference can occur between the UEs, from ST to PR and from PT to SR. Therefore, the following section will analyze how these interferences may occur and how we can mitigate them.

\section{TNFD INTER-USER INTERFERENCES AND MITIGATION}

In this section, we will first introduce how inter-user interferences can occur in TNFD communications when directional transmission is employed. Therefore, we will present our approach to suppress them by using an angle condition based on the beam of the employed antennas.

\section{A. Interference from $S T$ to $P R$}

Let us suppose that in Fig. 2, the BS transmits an RTSD frame to $U E_{7}$, which has no data packet to send to the BS, but after hearing ST-NCTS with its address the $U E_{8}$ becomes the ST, while $U E_{7}$ is the PR and BS is simultaneously the PT and SR. Let $\theta$ be the angle at $U E_{8}$ side formed by the lines 
$\overline{\left(\mathrm{UE}_{8}\right)\left(\mathrm{UE}_{7}\right)}$ and $\overline{\left(\mathrm{UE}_{8}\right)(\mathrm{BS})}$ shown in Fig. 2. Now, assuming that the value of the angle $\theta$ is very small and does not satisfy a given threshold value, such that the secondary transmission from $U E_{8}$ may cause interference to $U E_{7}$ reception. When the angle $\theta$ is very small it may not satisfy the minimum angle required to allow $U E_{8}$ to simultaneously transmit to the BS as ST in a TNFD communication scenario. Therefore, $U E_{8}$ may cause interference to $U E_{7}$ reception as depicted in Fig. 2 which shows the antenna beam from $U E_{8}$ toward BS passing through $U E_{7}$. Note that when the angle $\theta$ is higher than a given threshold value, the communication in TNFD scenario may occur without interference between users as it occurs between the BS, $U E_{1}$ and $U E_{2}$ in Fig. 2.

\section{B. Interference from $P T$ to $S R$}

Now, we assume that $U E_{2}$ transmits an RTSD control frame to the BS, which has no data packet to send to $U E_{2}$, but it has a packet for $U E_{1}$ therefore it transmits SR-NCTS control frame with the address of $U E_{1}$. In this case, $U E_{2}$ becomes the PT, the BS becomes simultaneously the PR and ST and $U E_{1}$ becomes the SR. Since the primary and the secondary transmissions occur simultaneously, $U E_{2}(\mathrm{PT})$ will cause interference to $U E_{1}$ reception (assuming that they are in the transmission range of each other), if the angle $\theta$ is lower than a given threshold value to allow ST to simultaneously transmit to the SR for TNFD communication scenario, interference will arise from the PT to the SR (from $U E_{2}$ to $U E_{1}$ ) similarly to the case of interference from ST to PR. Note that if $\theta$ is higher than a threshold value, the ongoing TNFD communication may not suffer from interference between users.

To mitigate these inter-user interferences in TNFD communications, we will establish some conditions referred to as TAC (Three-node Angle Condition) based on the transceiver directional antenna beams width and the angle between them at the ST side or the PT side.

\section{TNFD inter-user interferences mitigation}

In this subsection, we propose an efficient method to mitigate the inter-user interferences that occur when two adjacent UEs participate simultaneously in TNFD communication. Our approach is based on a condition called in this paper TAC as mentioned above. In other words, two UEs can participate simultaneously in TNFD communication when the separation angle between the locations of these UEs is sufficiently high than a threshold value at the PT side or the ST side depending on if the ongoing TNFD communication is source-based TNFD or destination-based TNFD. Before formulating TAC, we are interested to derive the angle (between the elements) that must be greater than a given threshold value. Fig. 3 a) shows an example of a TNFD communication scenario between the three following elements, $\mathrm{BS}, U E_{1}$ and $U E_{2}$, where $U E_{1}$ and $U E_{2}$ are assumed to be in the transmission range of each other. Moreover, in Fig. 3 a) are depicted the antennas beams $B^{B 1}$ (from BS toward $U E_{1}$ ), $B^{2 B}$ (from $U E_{2}$ toward $\mathrm{BS}$ ) and $B^{21}$ (from $U E_{2}$ toward $U E_{1}$ ), and their 
symmetric lines that point exactly on the desired direction. Observe that $\theta$ is the angle at the $U E_{2}$ side between the symmetric lines of the beams $B^{2 B}$ and $B^{21}$. As illustrated in Fig. 3 a), we assume that the BS has a data packet for $U E_{1}$, and $U E_{2}$ also has a data packet for the BS. Then, the BS can become PT or ST. If the BS initiates TNFD communication it becomes the PT and SR, $U E_{1}$ and $U E_{2}$ become the PR and the ST, respectively. If
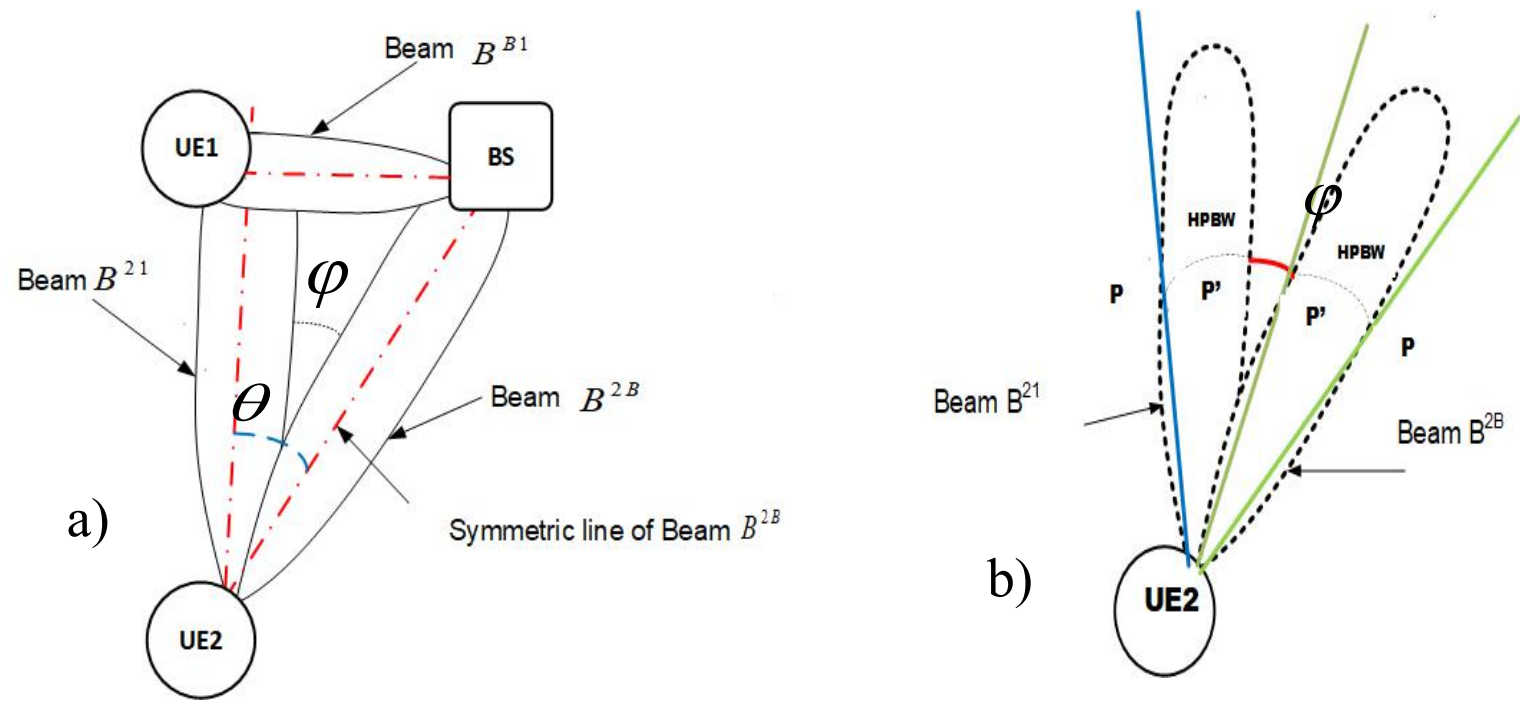

Fig. 3. A TNFD communication scenario: a) TAC verification scheme by estimating angle $\theta$ and b) angle $\varphi$ definition. $U E_{2}$ initiates a TNFD communication, it becomes the PT, then the BS become the PR and ST, while $U E_{1}$ becomes the SR. In both scenarios, the angle $\theta$ in Fig. 3 a) must be greater than a given threshold value in order to avoid inter-user interference from $U E_{2}$ to $U E_{1}$. Before selecting the $U E_{2}$ as the $\mathrm{ST}$ if $U E_{1}$ is the $\mathrm{PR}$ or before selecting $U E_{1}$ as the $\mathrm{SR}$ if $U E_{2}$ is the $\mathrm{PT}$, the BS must check whether, the angle $\theta$ between the symmetric lines of the beams $B^{2 B}$ and $B^{21}$ is greater than a given threshold value. The value of $\theta$ depends not only on the width of the beam of the employed antennas [34] but also on the value of $\varphi$ as shown in Fig. 3 a), and it is expressed as

$$
\theta=2 \beta+\varphi
$$

where $2 \beta$ is the width of the beam as mentioned above, and $\varphi$ is the angular separation of the beam $B^{2 B}$ and $B^{21}$ as shown in Fig. 3 a). Fig. 3 b) indicates how we can derive the angle $\varphi$, where $\varphi$ represents the angle between the two tangent lines at point $\mathrm{P}$ ' of the two beams $B^{2 B}$ and $B^{21}$, respectively. The reference point P' corresponds to the half-power bandwidth (HPBW) of each beam. When $\varphi$ is negative then the two beams may overlap and interference may occur if this ST or SR is selected to participate in the ongoing TNFD communication. The accurate choice of $\varphi$ depends on the radiation pattern of the used antennas system. If the gain of the main lobe reduces sharply increasing angular separation from the main lobe direction, then the value of $\varphi$ can be small. 
Now, we can announce the Three-node Angle Condition (TAC) as: The angle $\theta$ formed at the ST side between the antenna beam's symmetric lines $\overrightarrow{(\mathrm{ST})(\mathrm{SR})}$ and $\overrightarrow{(\mathrm{ST})(\mathrm{PR})}$, or the angle $\theta$ formed at PT side between the antenna beam's symmetric lines $\overline{(\mathrm{PT})(\mathrm{PR})}$ and $\overline{(\mathrm{PT})(\mathrm{SR})}$ must be greater than a threshold value to guarantee TNFD communication without interference. A neighboring user of a UE can simultaneously transmit or receive to or from the BS in TNFD communication if its position satisfies the TAC.

To avoid inter-user interference from PT to SR, the BS must always select an SR whose location satisfies the TAC. When there are many UEs whose locations satisfy the TAC, the BS can randomly select one of them before sending the SR-NCTS control frame with the address of the selected UE as the SR for the ongoing TNFD communication. To avoid interference from ST to PR, the BS must check first the TAC between the ST and the PR, and will only allocate the channel to an ST whose location satisfies the TAC. When there is more than one UEs whose positions satisfy the TAC, the BS will select one whose location form the highest angle $\theta$ for the PR. In the following subsection, we give the way to derive the angle $\theta$ at the BS side.

\section{Angle $\theta$ estimation}

In this sub-section, we present our approach to how BS can estimate $\theta$. Our approach is firstly based on the cellular user position estimation method based on the AOA (Angle of Arrival) presented in [35]. Then, we will apply mathematical cosine and sine rules to this method to derive the angle $\theta$. The method in [35] is based on the fact that when a BS receives a signal from a UE it can know its AOA and the corresponding coordinates. Because of noisy measurements in the UE position estimation, the authors in [35] proposed to collect information data from multiple base stations in order to resolve the ambiguities resulted from multiple crossings of the lines of position and to improve the positioning accuracy. Note that the information collection can be easily performed in the dense deployment scenario by allowing cooperation between different BSs.

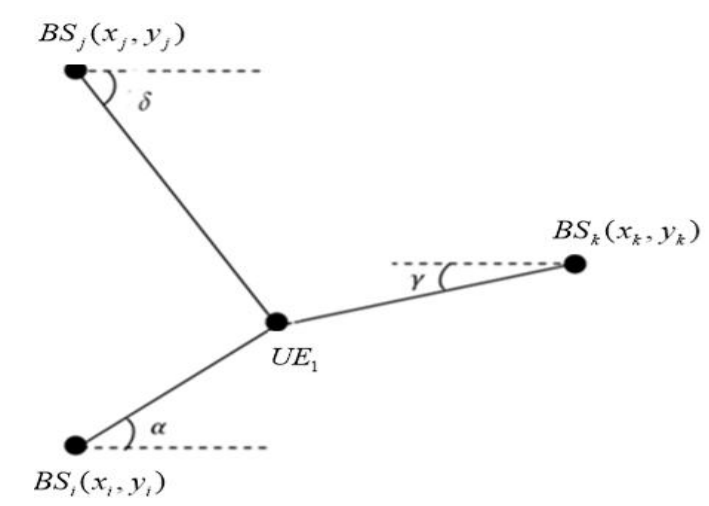

Fig. 4. Geometric layout of three angles of arrival approach [35]. 
In order to effectively avoid the interference in TNFD communications, the angle $\theta$ must be estimated with more exactitude, therefore we adopted the proposed method in [35]. The $U E_{1}$ can be simultaneously associated with many base stations. Fig. 4 shows the $U E_{1}$ and its association with the three closest base stations, $B S_{i}, B S_{j}$, and $B S_{k}$. The AOA at BS are $\alpha, \delta$, and $\gamma$ with respect to the base stations $B S_{i}, B S_{j}$, and $B S_{k}$, respectively. Given the values of the three angles $\alpha, \delta$, and $\gamma$, the $U E_{1}$ position can be determined by intersecting the three lines $\overrightarrow{B S}_{i} U E_{1}, \overrightarrow{B S}_{j} U E_{1}$ and $\overrightarrow{B S}_{k} U E_{1}$, and finding the simultaneous solution of the following three equations

Equation of $\overleftrightarrow{B S_{i} U E_{1}}$

$$
y=y_{i}+(\tan \alpha)\left(x-x_{i}\right)
$$

Equation of $\overleftrightarrow{B S_{j} U E_{1}}$

$$
y=y_{j}-(\tan \delta)\left(x-x_{j}\right)
$$

Equation of $\overparen{B S_{k} U E_{1}}$

$$
y=y_{k}+(\tan \gamma)\left(x-x_{k}\right)
$$

However, since the angles $\alpha, \delta$, and $\gamma$, subject to measurements errors, the three lines do not intersect at the same point. A reasonable method to estimate the UE position is to find the point of intersection of each two lines mutually and average the result.

Intersection of $\overrightarrow{B S_{i} U E_{1}}$ and $\overleftrightarrow{B S_{j} U E_{1}}$

$$
\begin{aligned}
& x_{a}=\frac{-y_{i}+y_{j}+(\tan \alpha) x_{i}+(\tan \delta) x_{j}}{\tan \alpha+\tan \delta} \\
& y_{a}=y_{i}+(\tan \alpha)\left(x-x_{i}\right)
\end{aligned}
$$

Intersection of $\overrightarrow{B S}_{i} U E_{1}$ and $\overleftrightarrow{B S_{k} U E_{1}}$

$$
\begin{gathered}
x_{b}=\frac{-y_{i}+y_{k}+(\tan \alpha) x_{i}-(\tan \gamma) x_{k}}{\tan \alpha-\tan \gamma} \\
y_{a}=y_{i}+(\tan \alpha)\left(x-x_{i}\right)
\end{gathered}
$$

Intersection of $\overleftrightarrow{B S_{j} U E_{1}}$ and $\overleftrightarrow{B S_{k} U E_{1}}$

$$
\begin{aligned}
& x_{c}=\frac{y_{j}-y_{k}+(\tan \delta) x_{j}+(\tan \gamma) x_{k}}{\tan \delta+\tan \gamma} \\
& y_{a}=y_{j}-(\tan \delta)\left(x-x_{j}\right)
\end{aligned}
$$

Thus, the position of $U E_{1}$ is

$$
\left(x_{1}, y_{1}\right)=\left(\frac{x_{a}+x_{b}+x_{c}}{3}, \frac{y_{a}+y_{b}+y_{c}}{3}\right)
$$


The position of $U E_{2}$ can be estimated similarly. Let $\left(x_{2}, y_{2}\right)$ be the position of $U E_{2}$. Now, with these accurate user positions, the value of angle $\theta$ can be estimated. Fig. 5 shows the positions of $U E_{1}$ and $U E_{2}$ with respect to the BS and their angles $\phi_{1}$ and $\phi_{2}$, respectively. Also, Fig. 5 shows a TNFD communication scenario between $\mathrm{BS}, U E_{1}$ and $U E_{2}$. Assume that $U E_{1}$ is $\mathrm{PR}, U E_{2}$ is $\mathrm{ST}$ and the $\mathrm{BS}$ is simultaneously PT and SR. Now, we can apply mathematical cosine and sine rules to the triangle represented in Fig. 5 to derive the angle $\theta$.

The angles $\phi_{1}$ and $\phi_{2}$ can be calculated as $\phi_{1}=\tan ^{-1} \frac{x_{1}}{d_{1}}$, and $\phi_{2}=\tan ^{-1} \frac{x_{2}}{d_{2}}$. Then, we can obtain the angle $\phi$ as $\phi=\phi_{2}-\phi_{1}$. Let be $d_{1}$, the distance from the BS to $U E_{1}$, which is calculated as $d_{1}=\sqrt{x_{1}^{2}+y_{1}^{2}}$,

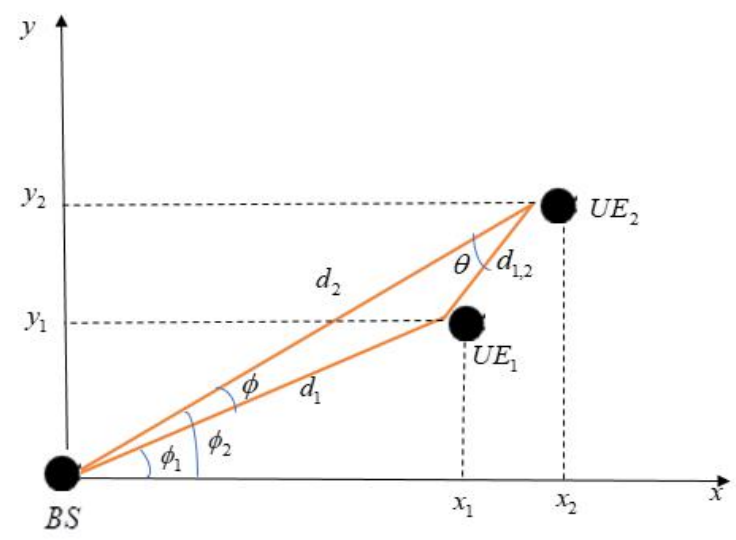

Fig. 5. Estimation of angle $\theta$ by using cosine and sine rules.

$d_{2}$ is the distance from the BS to $U E_{2}, d_{2}=\sqrt{x_{2}{ }^{2}+y_{2}{ }^{2}}$ and $d_{1,2}$ is the distance from $U E_{1}$ to $U E_{2}$, which can be derived by using the law of cosine theorem, as

$$
\begin{aligned}
& d_{1,2}^{2}=d_{1}^{2}+d_{2}^{2}-2 d_{1} d_{2} \cos \phi \\
& d_{1,2}=\sqrt{d_{1}^{2}+d_{2}^{2}-2 d_{1} d_{2} \cos \phi}
\end{aligned}
$$

By using the law of sine, we can write

$$
\begin{aligned}
& \sin \theta=\frac{d_{1} \sin \phi}{d_{1,2}} \\
& \theta=\sin ^{-1}\left(\frac{d_{1} \sin \phi}{d_{1,2}}\right)
\end{aligned}
$$




\section{WCFD-MAC PROTOCOL DESIGN}

Our MAC design is based on the IEEE 802.11 standard [36] and HFD-MAC protocol presented in [10]. Therefore, some control frames are the same as those presented in [10] and IEEE 802.11 standard. Furthermore, the CSMA/CA backoff algorithm is the same as in [10], [36]. The control frames used in this paper are RTSD, CTSD, RTSC, SR-NCTS, ST-NCTS, and ACK. Their formats are shown in Fig. 6. The ACK frame is the same as the standard of IEEE 802.11, then it is not shown in Fig. 6.

The main differences with the one presented in [10] are:

- Our design uses omnidirectional and directional antenna transmission, instead of omnidirectional only.

- our design allows two adjacent UEs to simultaneously transmit and receive to and from the BS in TNFD communication, which is impossible in [10].

- Instead of performing a self-timer algorithm by the candidates UEs to become the ST after hearing the

\begin{tabular}{|c|c|c|c|c|c|c|}
\hline $\begin{array}{l}\text { RTSD Frame } \\
20.25 \text { bytes }\end{array}$ & $\begin{array}{c}\text { Frame } \\
\text { Control (2) }\end{array}$ & $\begin{array}{c}\text { Duration } \\
\text { (2) }\end{array}$ & $\begin{array}{l}\text { RA } \\
(6)\end{array}$ & $\begin{array}{l}\text { TA } \\
(6)\end{array}$ & $\begin{array}{c}\text { DI } \\
\text { (2 bits) }\end{array}$ & $\begin{array}{l}\text { CRC } \\
\text { (4) }\end{array}$ \\
\hline $\begin{array}{l}\text { RTSC Frame } \\
26 \text { bytes }\end{array}$ & $\begin{array}{c}\text { Frame } \\
\text { Control (2) }\end{array}$ & $\begin{array}{l}\text { Duration } \\
\text { (2) }\end{array}$ & $\begin{array}{l}\text { RA } \\
\text { (6) }\end{array}$ & $\begin{array}{l}\text { IRA } \\
(6)\end{array}$ & $\begin{array}{l}\text { TA } \\
\text { (6) }\end{array}$ & $\begin{array}{l}\text { CRC } \\
\text { (4) }\end{array}$ \\
\hline $\begin{array}{l}\text { CTSD Frame } \\
14.25 \text { bytes }\end{array}$ & $\begin{array}{c}\text { Frame } \\
\text { Control (2) }\end{array}$ & $\begin{array}{c}\text { Duration } \\
\text { (2) }\end{array}$ & $\begin{array}{l}\text { RA } \\
\text { (6) }\end{array}$ & $\begin{array}{c}\text { DI } \\
\text { (2 bits) }\end{array}$ & $\begin{array}{l}\text { CRC } \\
\text { (4) }\end{array}$ & \\
\hline $\begin{array}{c}\text { SR-NCTS Frame } \\
20 \text { bytes }\end{array}$ & $\begin{array}{c}\text { Frame } \\
\text { Control (2) }\end{array}$ & $\begin{array}{c}\text { Duration } \\
\text { (2) }\end{array}$ & $\begin{array}{l}\text { PRA } \\
\text { (6) }\end{array}$ & $\begin{array}{l}\text { SRA } \\
(6)\end{array}$ & $\begin{array}{l}\text { CRC } \\
\text { (4) }\end{array}$ & \\
\hline \multirow[t]{2}{*}{$\begin{array}{l}\text { ST-NCTS Frame } \\
20.25 \text { bytes }\end{array}$} & $\begin{array}{c}\text { Frame } \\
\text { Control (2) }\end{array}$ & $\begin{array}{l}\text { Duration } \\
\text { (2) }\end{array}$ & $\begin{array}{l}\text { STA } \\
\text { (6) }\end{array}$ & $\begin{array}{l}\text { PTA } \\
\text { (6) }\end{array}$ & $\begin{array}{c}\text { DI } \\
\text { (2 bits) }\end{array}$ & $\begin{array}{l}\text { CRC } \\
\text { (4) }\end{array}$ \\
\hline & \multicolumn{6}{|c|}{$\begin{array}{l}\text { RA: Receiver Address } \\
\text { TA: Transmitter Address } \\
\text { PRA: Primary Receiver Address } \\
\text { PTA: Primary Transmitter Address } \\
\text { SRA: Second Receiver Address } \\
\text { STA: Secondary Transmitter Address } \\
\text { IRA: Intermediate Receiver Address } \\
\text { STA: Secondary Transmitter Address } \\
\text { DI: Duplexing Indicator }\end{array}$} \\
\hline
\end{tabular}

Fig. 6. Control frames formats of CFD-MAC design.

NDI control frame in source-based TNFD scenario as occur in [10], in our design the BS will immediately select one of these UEs and then transmit ST-NCTS frame with its address.

- A new HD scheme is proposed (TNHD) in our MAC design, which can enhance the throughput of UEs located at the small cell border. 
The meaning of DI bits is the same as those in [10] and are presented in Table I.

TABLE I. DESCRIPTION OF DUPLEXING INDICATOR
\begin{tabular}{|c|c|}
\hline Value & Meaning of DI \\
\hline 00 & Not used \\
01 & Sender can Rx only \\
10 & Sender can Tx only \\
11 & Sender can Tx an Rx simultaneously \\
\hline
\end{tabular}

The communications between the BS and UEs may be BFD, TNFD, and HD, depending on if the transmitter and receiver have a data packet to transmit to each other or not, as explained above. When a PT has data to send to a PR, it first waits for the channel to become idle for DIFS duration, then it transmits omnidirectionally an RTSD frame to the PR. The communication can become BFD, TNFD, HD, or TNHD depending on the reply of the PR to the PT.

According to the location and traffic condition at FD UEs involved in this communication scenario, four possible cases arise as depicted in Fig. 7: BFD, TNFD (destination-based TNFD and sourcebased TNFD), HD, and TNHD.

\section{A. Bidirectional FD communication (BFD)}

After receiving an RTSD frame from BS, if a UE also has a data packet for BS, it replies with a CTSD frame with a DI value of 11 . Then, the communication becomes bidirectional, and both BS and UE transmit simultaneously their data to each other after exchanging RTSD/CTSD control frames. After a SIFS time, the ACK frames are transmitted to each other simultaneously as the data packets. In this case, the BS becomes at PT/SR and the corresponding UE becomes PR/ST simultaneously. Note that communication can also be initiated by a UE.

a)

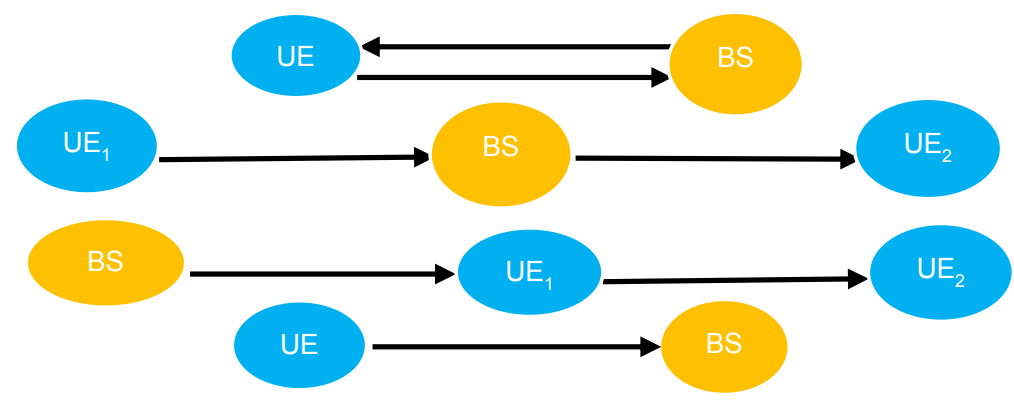

Fig. 7. Different communication schemes: a) BFD communication, b) TNFD communication, c) TNHD communication, d) HD communication.

\section{B. $\quad$ Three-node FD communication (TNFD)}

The TNFD communication schemes can be classified into a destination-based TNFD and sourcebased TNFD. The destination-based TNFD can occur when a UE initiates the transmission to the BS while the source-based TNFD can occur when the BS initiates the transmission to a UE. 
-Destination-based TNFD communication: when a UE (PT) initiates the transmission to the BS (PR); if the PR does not have a data packet to the PT, however, it has a data packet for another UE, thus the PR becomes ST and the receiver of its data packet becomes SR. This scenario is referred to as destination-based three nodes FD. The PR/ST replies with the SR-NCTS frame where it will indicate the address of an SR whose position satisfies the TAC with respect PT in the SR address field as shown in Fig. 6. The SR-NCTS frame will inform both PT and SR about their data exchange information as

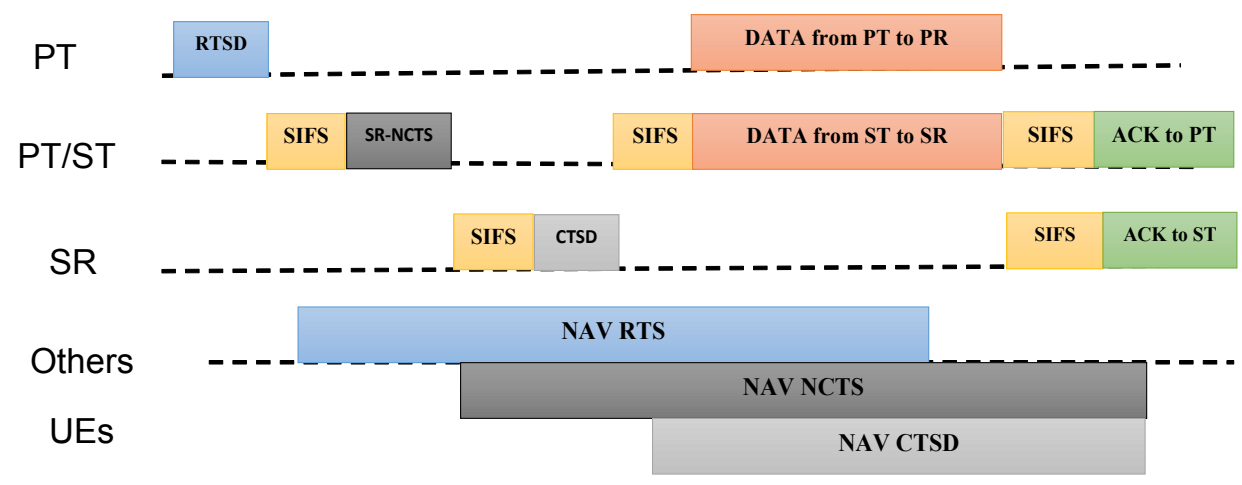

Fig. 8. Destination-based TNFD communication scheme.

proposed in [10]. After receiving the SR-NCTS, the SR replies with another CTS control frame to the $\mathrm{PR} / \mathrm{ST}$, and the data packet exchanges will occur between the three network elements after a SIFS time. Note that, the SR must be a UE whose location satisfies the TAC which can reply to PR/ST and TNFD communication will occur even if they are in the transmission range of each other thanks to directional transmission unlike the MAC protocol proposed in [10], [37]. After the data transmission, ACK frames are transmitted directionally and simultaneously from the PR to the PT and from the SR to the ST.

However, if the PR (BS) does not receive the SR-NCTS from SR, it will receive the data packet from PT after SIFS plus CTS times. Other nodes update their NAV times according to the time defined by the control frames. This case is shown in Fig. 8.

-Source-based TNFD communication: after receiving an RTSD frame from BS (PT), if the corresponding UE (PR) does not have any data packet to send to PT, it will transmit CTSD with a DI value of 01 . When PT receives the CTSD frame, it will try to find another UE to become the ST. The selected ST must be a UE whose position satisfies the TAC with respect to the PR. The PT (BS) will immediately select one of the UEs whose position satisfies the TAC with respect to the PR. The ST selection can be random or based on the value of $\theta$. For example, the ST can be a UE whose position form the highest value of the angle $\theta$ with respect to the PR. After selecting an ST, the PT will transmit the ST-NCTS control frame with the address of the selected ST in the STA field. Then, 
communication becomes source-based TNFD. After transmitting the ST-NCTS control frame with the address of the selected ST, the data packets exchanges will occur between the three network elements after a SIFS time. Note that, if there is no UE whose position satisfies the TAC to become the ST, thus, HD communication will occur. The ACKs frames are transmitted directionally after the data packets exchanges and a SIFS time from the PR to the PT (BS) and from the SR (BS) to the ST. This case is shown in Fig. 9.

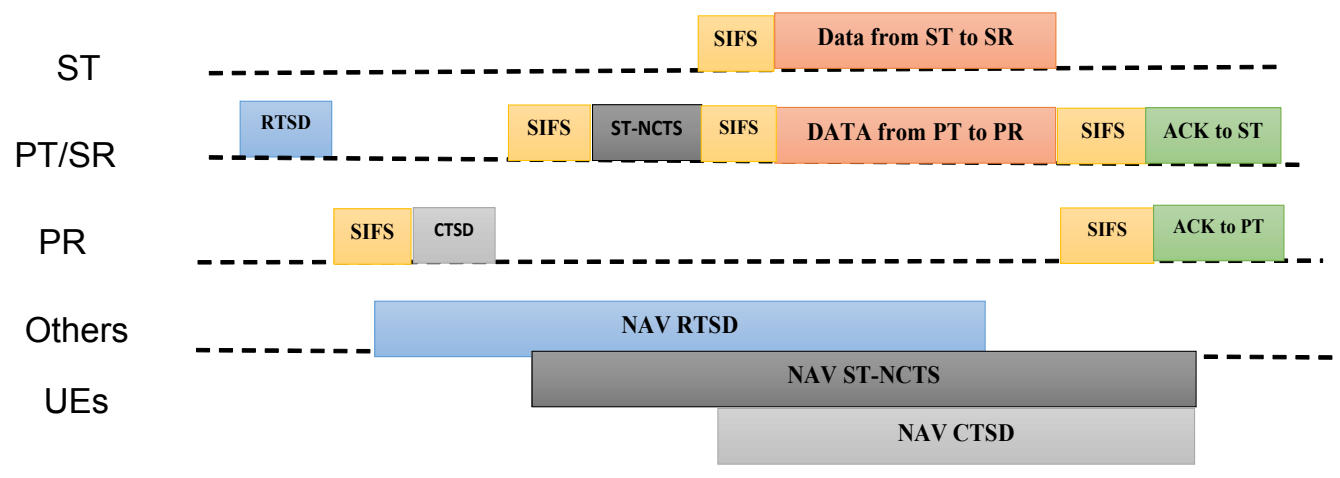

Fig. 9. Source-based TNFD communication scheme.

\section{HD communication}

HD communication will occur in the following scenario: i) after receiving an RTSD frame from BS (PT), if the receiving UE (PR) has no data packet for PT, it will reply with a CTSD frame with a DI value of 01 . The PT will try to find an ST which satisfies the TAC. If it does not find any UE able to satisfy the TAC, HD communication will occur, ii) after transmitting an RTSD frame by a UE (PT) to the BS (PR), HD communication will occur if the PR has no data packet to send to another UE including the PR, or if it has a data packet to send to UEs whose location cannot satisfy the TAC. The packet exchange will occur after SIFS time. Also, the ACK frame is transmitted after a SIFS time.

\section{Three-node HD communication (TNHD)}

When the BS (PT) transmits an RTSD to a UE (PR) located at the border of the covered small cell which has no data packet for BS, and the BS does not find any ST satisfying the TAC, then HD communication will occur. In this scenario as the UE is far from the BS, to maximize its throughput the BS will transmit the RTSC control frame containing the address of an intermediate UE and the PT address. After hearing this control frame, the intermediate UE will transmit the SR-NCTS frame with its address and the address of the PT. When the PT hears this frame, it will steer its directional antenna beam toward the intermediate UE. After a SIFS time, the BS will send the data packet to the intermediate UE, which can forward it to the cell boundary UE. After a SIFS time, ACK frames are transmitted from the cell boundary UE to the intermediate UE, and from the intermediate UE to the BS. Note that in this three-node communication scheme, only one data packet is transmitted, instead of two, therefore, it is a special case of HD communication. This scenario is shown in Fig. 10. 


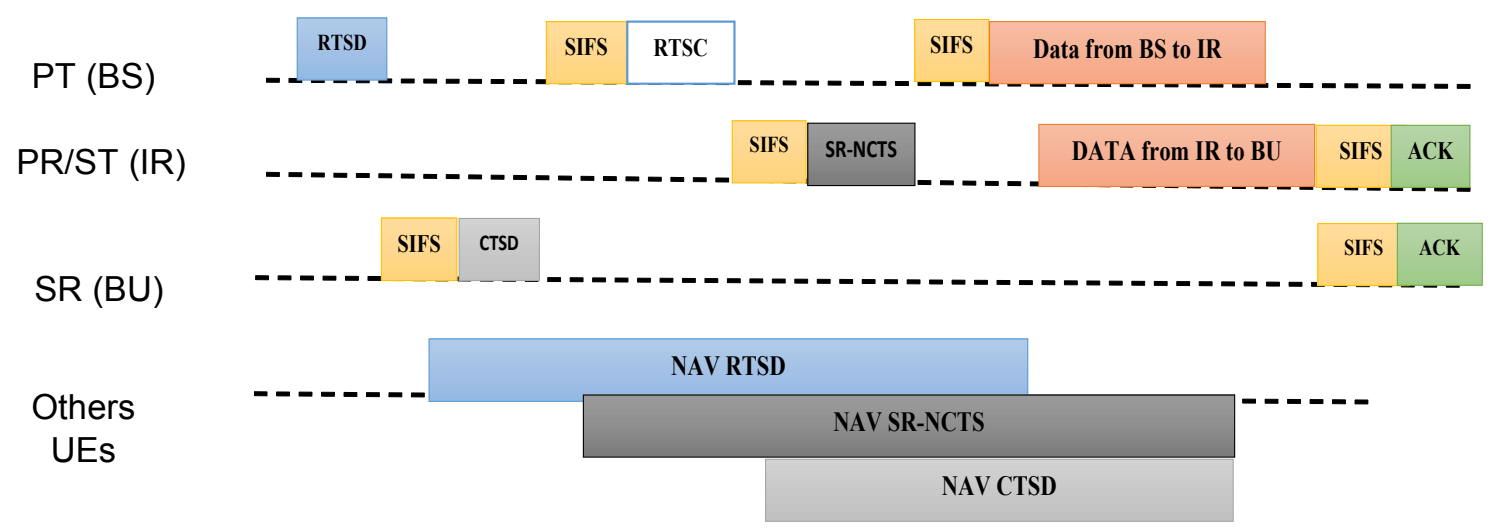

Fig. 10. TNHD communication scheme.

\section{E. Computational complexity}

In this paper, our design considers directional transmission, which is performed by the beamforming technique and AoA estimation. The AoA estimation algorithm must have high resolution and low computational complexity. Our design can use the algorithm proposed in [38] since it is computationally more efficient and it doesn't require an exhaustive search through all possible steering vectors for AOA estimation. The computational complexity of this algorithm was derived in [39]. Additionally, our design follows the user scheduling algorithm based on CSMA/CA and RTS/CTS mechanisms of the IEEE 802.11 standard, thus having the same computational complexity as this standard.

\section{PERFORMANCE EVALUATION}

In this section, the performance of our MAC protocol design is evaluated through simulations based on Bianchi's model [40] and the transmission probability derivation method presented in [10]. To this end, we will present in the first subsection, transmission probability derivation, then we will present throughput analytical expression derivation after the impact of SI cancellation consideration, and finally, we will present simulation results.

\section{A. Transmission probability}

To derive the probability equations in this paper, our approach follows the method developed by Alim et al [10] based on the Poisson process and the assumption of the M/M/1 system model. Thus, different expressions of transmission probability corresponding to BFD, TNFD, and HD communications are obtained by using the packet arrival rate (PAR) at the $\mathrm{BS}$ and packet generation rate (PGR) at UEs.

Let $\lambda_{B U}$ and $\lambda_{U B}$ be the PAR at BS from the core network to all the UEs, and PGR at each UE, respectively. The PAR at the BS from the core network and $n$ UEs (where $n$ is the number of UEs) is

$$
\lambda_{\text {total }}=n \lambda_{B U}+n \lambda_{U B}
$$


Let $T_{w}$ be the average waiting time ( $T w$ ) for a packet in the system queue, and it is expressed as

$$
T w=\frac{\text { arrivalrate }}{\text { servicerate(servicerate-arrivalrate })}=\frac{\lambda_{\text {iotal }}}{\mu\left(\mu-\lambda_{\text {iotal }}\right)}
$$

where $\mu$ is the average data rate of the BS.

In the following subsections, we will present the transmission probability expressions for BFD, TNFD, HD.

\section{Bidirectional FD transmission probability}

Independently from which network element initiates the transmission (BS or UE), BFD transmission can occur. Therefore, we can identify two scenarios for BFD communication to obtain the BFD transmission probability $\left(P_{B F D}\right)$.

Scenario 1 (BS initiates the communication): when the BS begins the communication by sending an RTSD control frame to a UE, if this UE has a data packet to send to the BS at the same time, thus, BFD transmission will occur between them. To obtain $P_{B F D}$ corresponding to scenario 1 , we make the following observations related to the conditional probability: i) the conditional probability that the packet arriving at the BS is for a UE, which is $\frac{n \lambda_{B U}}{\lambda_{\text {total }}}$; ii) the probability that the corresponding UE also has a packet to send to the BS within the time $T_{1}$ is $\left(1-e^{-\lambda_{U B} T_{1}}\right)$, where $T_{1}=T_{\text {RTSD }}+T_{S I F S}+T_{w}$, and the meaning of $T_{R T S D}$ and $T_{S I F S}$ are given in Table II.

Scenario 2 (UE initiates the communication): when a UE begins the communication by sending an RTSD control frame to the BS, only if the BS has a data packet to send to the corresponding UE at the same time, BFD transmission will occur between them. To obtain $P_{B F D}$ corresponding to scenario 2, we make the following observations related to the probability theory: i) the conditional probability that a packet arriving at BS is sent by a UE is $\frac{n \lambda_{U B}}{\lambda_{\text {total }}}$; ii) the probability that BS also has at least one packet to send to the corresponding UE within the time $T_{1}$ is $\left(1-e^{-\lambda_{B U} T_{1}}\right)$. Now, we can write BFD transmission probability $P_{B F D}$ corresponding to two scenarios presented above as

$$
P_{B F D}=\frac{n \lambda_{B U}}{\lambda_{\text {total }}}\left(1-e^{-\lambda_{U B} T_{1}}\right)+\frac{n \lambda_{U B}}{\lambda_{\text {total }}}\left(1-e^{-\lambda_{B U} T_{1}}\right)
$$

\section{Three-node FD transmission probability}

TNFD communication can occur whether the BS initiates the communication. In this case, we can also identify two scenarios for TNFD communication in order to obtain the TNFD transmission probability $\left(P_{T N F D}\right)$. 
Scenario 1 (UE initiates the communication): when a UE transmits an RTSD control frame to the BS, TNFD communication can occur depending on some conditions that must be satisfied. The first of these conditions is, "the BS has no data packet to send to the corresponding UE". The second condition is, "the BS has a data packet to send to another candidate UE that satisfies the TAC". To obtain $P_{T N F D}$ corresponding to the scenario 1, we make the following observations related to the probability theory: i) the probability that a packet arriving at the BS is by a UE is $\frac{n \lambda_{U B}}{\lambda_{\text {total }}}$; ii) the probability that the BS has no data packet to send to the corresponding UE within the time $T_{1}$ is $\left(e^{-\lambda_{B U} T_{1}}\right)$; iii) the probability that the BS has at least a data packet to send to another UE which satisfy the TAC within the time $T_{1}$ is $\left(1-e^{-(n-1) \lambda_{B U} T_{1}}\right) \gamma$, where $\gamma(0 \leq \gamma \leq 1)$ is the proportion of UEs that satisfy the TAC.

Scenario 2 (BS initiates the communication): when the BS begins the transmission by sending an RTSD to a UE, TNFD communication can occur only if some conditions are satisfied. The first condition that must be satisfied is "the corresponding UE has no data packet in its buffer to send to the BS'. The second condition is "another UE has a data packet to send to the BS at the same time". To obtain $P_{T N F D}$ corresponding to the scenario 2, we make the following observations related to the probability theory: i) the probability that the BS at least has a data packet to send to a UE is $\frac{n \lambda_{B U}}{\lambda_{\text {total }}}$; ii) the probability expression that the corresponding UE has no data packet to send to the BS within the time $T_{1}$ is $\left(e^{-\lambda_{C B} T_{1}}\right)$; iii) the probability that another UE that satisfies the TAC has a data packet for the BS within time $T_{2}$ is $\left(1-e^{-(n-1) \lambda_{U B} \gamma T_{2}}\right)$, where $T_{2}=T_{R T S D}+2 T_{\text {SIFS }}+T_{C T S D}+T_{S T-N C T S}+T_{w}$, and the meaning of $T_{C T S D}$ and $T_{S T-N C T S}$ are given in Table II.

Now, we can write TNFD transmission probability $P_{T N F D}$ corresponding to the two scenarios presented above is expressed as

$$
P_{T N F D}=\frac{n \lambda_{U B}}{\lambda_{\text {total }}}\left(e^{-\lambda_{B U} T_{1}}\right)\left(1-e^{-(n-1) \lambda_{B U} T_{1}}\right) \gamma+\frac{n \lambda_{B U}}{\lambda_{\text {total }}}\left(e^{-\lambda_{U B} T_{1}}\right)\left(1-e^{-(n-1) \lambda_{U B} \gamma T_{2}}\right)
$$

\section{HD transmission probability}

HD communication can occur whether the BS initiates the communication. Here, we can also identify three scenarios for HD communication to derive the HD transmission probability $\left(P_{H D}\right)$.

Scenario 1 (UE initiates communication): when a UE begins the transmission to a UE in the covered cell, HD communication can occur if some conditions are satisfied. The first of these conditions is "the BS has no data packet to send to any UE including the corresponding UE". The second condition is "the BS has a data packet to send to another UE that cannot satisfy the TAC". To obtain $P_{T N F D}$ corresponding to the scenario 1 , we make the following observations related to the probability 
theory: i) the probability that a UE initiates the transmission and the probability that the BS has no data to another UE including the corresponding UE is $\frac{n \lambda_{U B}}{\lambda_{\text {total }}}\left(e^{-n \lambda_{U B} T_{1}}\right)$; ii) the probability that after initiating the transmission by a UE, the BS has no data to send to the corresponding UE within time $T_{1}$ and the BS has at least one packet to send to another UE that cannot satisfy the TAC within time $T_{1}$ is $\frac{n \lambda_{U B}}{\lambda_{\text {total }}}\left(e^{-\lambda_{B U} T_{1}}\right)\left(1-\left(e^{-(n-1) \lambda_{B U} T_{1}}\right)(1-\gamma)\right.$.

Scenario 2 (BS initiates communication): HD communication can occur when the BS begins the communication to a UE if some conditions are satisfied. The first of these conditions is "the UE receiver of the RTSD control frame (corresponding UE) has no data packet to send to the transmitter of the RTSD control frame (BS)". The second condition is " another UE that satisfies the TAC has no data packet to send to the BS',. To obtain $P_{H D}$ corresponding to scenario 2, we make the following observations related to the probability theory: i) the probability that the BS has a packet for a UE is $\frac{n \lambda_{B U}}{\lambda_{\text {total }}}$; ii) the probability that the corresponding UE has no data packet to send to the BS within time $T_{1}$, is $\left(e^{-\lambda_{U B} T_{1}}\right)$; iii) the probability that another UE that satisfies the TAC has no data to send to the BS within time $T_{2}$ is $\left(e^{-(n-1) \lambda_{U B} \gamma T_{2}}\right)$.

Scenario 3 (TNHD communication when BS initiates communication): when the BS initiates the communication, TNHD communication will occur if the corresponding UE is located at the border of the covered small cell and it has no data packet to send to the BS, and any other UE whose position satisfies the TAC has no data packet to send to the BS. To derive $P_{T N F D}$ corresponding to this case, we make the following observations related to the probability theory: i) the probability that the BS has a data packet for a UE that is located at the cell boundary is $\frac{n \lambda_{B U}}{\lambda_{\text {total }}} \sigma$, where $\sigma(0 \leq \sigma \leq 1)$ is the proportion of UEs at cell border; ii) the probability that the corresponding UE has no data packet to send to the BS within time $T_{1}$ is $\left(e^{-n \lambda_{U_{B}} T_{1}}\right) \sigma$; iii) the probability that any other UE whose position satisfies the TAC has no data to send to the BS within time $T_{2}$, this is $\left(e^{-(n-1) \lambda_{V_{B} B T_{2}}}\right)$

Now, we can write HD transmission probability $P_{H D}$ corresponding to the three scenarios presented above is expressed as

$$
\begin{aligned}
P_{H D} & =\frac{n \lambda_{U B}}{\lambda_{\text {total }}}\left(e^{-n \lambda_{U B} T_{1}}\right)+\frac{n \lambda_{U B}}{\lambda_{\text {total }}}\left(e^{-\lambda_{B U} T_{1}}\right)\left(1-\left(e^{-(n-1) \lambda_{B U} T_{1}}\right)(1-\gamma)\right. \\
+ & \frac{n \lambda_{B U}}{\lambda_{\text {total }}}\left(e^{-\lambda_{U B} T_{1}}\right)\left(e^{-(n-1) \lambda_{U B} \gamma T_{2}}\right)+\frac{n \lambda_{B U}}{\lambda_{\text {total }}} \sigma\left(e^{-n \lambda_{U B} T_{1}}\right) \sigma\left(e^{-(n-1) \lambda_{U B} Y T_{2}}\right)
\end{aligned}
$$




\section{B. Impact of SI cancellation coefficient}

The SI cancellation performance depends on several factors, such as system bandwidth, antenna displacement error, and transmit signal amplitude difference [24], and channel estimation error at the receiver side and active/passive cancellation robustness.

Let $E_{H}, E_{B}$ and $E_{T}$ be the effective packet payloads of HD, BFD and TFD transmission, respectively. Their expressions are given by

$$
E=\left\{\begin{array}{c}
E_{B}=k\left(E_{1}+E_{2}\right) \\
E_{T}=k E+E_{2} \\
E_{H}=\frac{E_{1}+E_{2}}{2}
\end{array}\right.
$$

where $E_{1}$ and $E_{2}$ are the packet payloads transmitted in both directions between the BS and one UE in BFD transmission, or packet payload exchanged during a TNFD transmission between the BS and two other UEs. $k(0 \leq k \leq 1)$ denotes the SI cancellation coefficient. When $k$ approaches 0 , this means that the SI causes large interference on FD communication. When $k$ approaches 1 , it implies that the SI causes little interference on FD communication. Note that the differentiation between the effective packet payloads corresponding respectively to BFD and TNFD transmissions is due to the fact that BFD links are more affected by the SI than TNFD links [41], since in BFD scheme each network element involved in the communication has to mitigate SI, while in TNFD scenario, only one network element (BS) has to mitigate SI.

\section{Throughput expression}

To derive the throughput analytical expression for our design, our approach is based on Bianchi's model [40] which was developed for HD communication scenarios. From Bianchi's throughput model derived in [40], the system throughput can be expressed as

$$
S=\frac{P_{H D} E_{H}+P_{B F D} E_{B}+P_{T N F D} E_{T}}{\left(P_{H D}+P_{B F D}+P_{T N F D}\right) T_{S}+P_{C} T_{C}+P_{i d l} T_{\text {slot }}}
$$

where $P_{i d l}=1-P_{t r}, P_{t r}$ denotes the probability that there is at least one transmission in the considered slot time and it depends on the parameters of exponential backoff algorithm (minimum and maximum backoff window size) and of the number of nodes in the covered small cell and can be obtained as in [40]. The meaning of all the parameters in (18) are reported in Table II. $T_{s}$ and $T_{c}$ in (19) are respectively expressed as follows

$$
\begin{gathered}
T_{s}=T_{D I F S}+T_{R T S D}+T_{C T S D}+T_{d}+3 T_{S I F S}+T_{A C K}+4 T_{P} \\
T_{c}=T_{D I F S}+T_{R T S D}+T_{p}
\end{gathered}
$$

The collisions can occur only on the RTSD frames in the systems where each packet is transmitted through the RTSD/CTSD access mechanism [40]. 
TABLE II. MEANING OF PARAMETERS

\begin{tabular}{|l|l|}
\hline Parameter & Description \\
\hline$T_{A C K}$ & MAC-layer ACK frame transmission time \\
\hline$T_{R T S D}$ & RTSD frame transmission time \\
\hline$T_{C T S D}$ & CTSD frame transmission time \\
\hline$T_{S R-N C T S}$ & SR-NCTS frame transmission time \\
\hline$T_{S I F S}$ & Short Inter-Frame Space time \\
\hline$T_{S T-N C T S}$ & ST-NCTS frame transmission time \\
\hline$T_{D I F S}$ & DCF Inter-Frame Space \\
\hline$T_{p}$ & Propagation time over the air \\
\hline$T_{s l o t}$ & MAC-layer Slot duration time \\
\hline$T_{c}$ & Average collision time \\
\hline$T_{s}$ & Data packet transmission time \\
\hline$T_{d}$ & \\
\hline
\end{tabular}

Now, assuming that, in both transmission directions all the packet payloads have the same length, $E_{1}=E_{2}=E$, then each effective packet payloads $E_{B}, E_{T}$ and $E_{H}$ in (17) becomes $E_{B}=2 k E$, $E_{T}=(k+1) E$ and $E_{H}=E$, respectively. Furthermore, assuming perfect SI cancellation $(k=1)$, the system throughput expression becomes

$$
S=\frac{\left(P_{H D}+2 P_{B F D}+2 P_{T N F D}\right) E}{\left(P_{H D}+P_{B F D}+P_{T N F D}\right) T_{S}+P_{C} T_{C}+P_{i d l} T_{s l o t}}
$$

Let $\omega(0 \leq \omega \leq 1)$ be the proportion of occurrence of FD communications (including both, BFD and TNFD) in the cell. Assuming also a perfect SI cancellation, the system throughput expression becomes

$$
S=\frac{\left((1-\omega) P_{H D}+2 \omega\left(P_{B F D}+2 P_{T N F D}\right)\right) E}{\left(P_{H D}+P_{B F D}+P_{T N F D}\right) T_{S}+P_{C} T_{C}+P_{\text {idl }} T_{\text {slot }}}
$$

Now, when the SI cancellation coefficient $k \neq 0$, the system throughput becomes

$$
S=\frac{P_{H D} E+2 k P_{B F D} E+(k+1) P_{T N F D} E}{\left(P_{H D}+P_{B F D}+P_{T N F D}\right) T_{S}+P_{C} T_{C}+P_{i d l} T_{s l o t}}
$$


D. Simulation results

In this subsection, we present simulation results of WCFD-MAC protocol design. In the simulation stage, some assumptions have been performed. We assumed that TAC is satisfied with the sourcebased TNFD scenario when the angle $\theta_{1}$ between the straight lines $\overrightarrow{(B S)(P R)}$ and $\overrightarrow{(B S)(S T)}$ is greater than a given threshold value. Similarly, we assumed that TAC is satisfied with the destination-based TNFD scenario when the angle $\theta_{2}$ between the straight lines $\overrightarrow{(B S)(S R)}$ and $\overrightarrow{(B S)(P T)}$ is greater than a given threshold value. We evaluated our method by performing simulations under non-saturation conditions, because in saturation conditions all the communications in the covered small cell may be BFD communications. Note that, saturation conditions assume that, always all the UEs and BS have a data

TABLE III. SIMULATION PARAMETERS

\begin{tabular}{ll}
\hline Parameter & value \\
\hline Payload size & 2000 bytes \\
\hline RTSD & 20.25 bytes \\
\hline CTSD & 14.25 bytes \\
\hline RTSC & 26 bytes \\
\hline ACK & 14 bytes \\
\hline SR-NCTS & 20 bytes \\
\hline ST-NCTS & 20.25 bytes \\
\hline Data rate & $54 \mathrm{Mbits} / \mathrm{s}$ \\
\hline Control frames rate & $6 \mathrm{Mbits} / \mathrm{s}$ \\
\hline Slot time & $9 \mu \mathrm{s}$ \\
\hline SIFS time & $16 \mu \mathrm{s}$ \\
\hline DIFS time & $34 \mu \mathrm{s}$ \\
\hline CWmin & 15 \\
\hline PLCP preamble duration & $16 \mu \mathrm{s}$ \\
\hline PLCP header duration & $4 \mu \mathrm{s}$ \\
\hline Propagation time over the air & $1 \mu \mathrm{s}$ \\
\hline
\end{tabular}

packet to each other. For all the simulation scenarios in this paper, the PAR at the BS and the PGR at the UEs were fixed to 50 Packets/s and 5 packets/s, respectively. We compare our simulation results with the previous works related to FD MAC protocol, particularly, with those obtained in [10], [24], [36], [42]. We selected [10] for comparison since it is the only work that addressed partially inter-user interference in TNFD communication for FD MAC protocol according to our acknowledgment. Thus, for a fair comparison, we used the same simulation parameters as in [10]. Additionally, we compare our simulation results with those obtained in [24], because it is the most cited work on FD MAC protocol design for $5 \mathrm{G}$ cellular networks in literature. These simulation parameters are shown in Table III. To evaluate the performance of our design, the following aspects have been evaluated: 


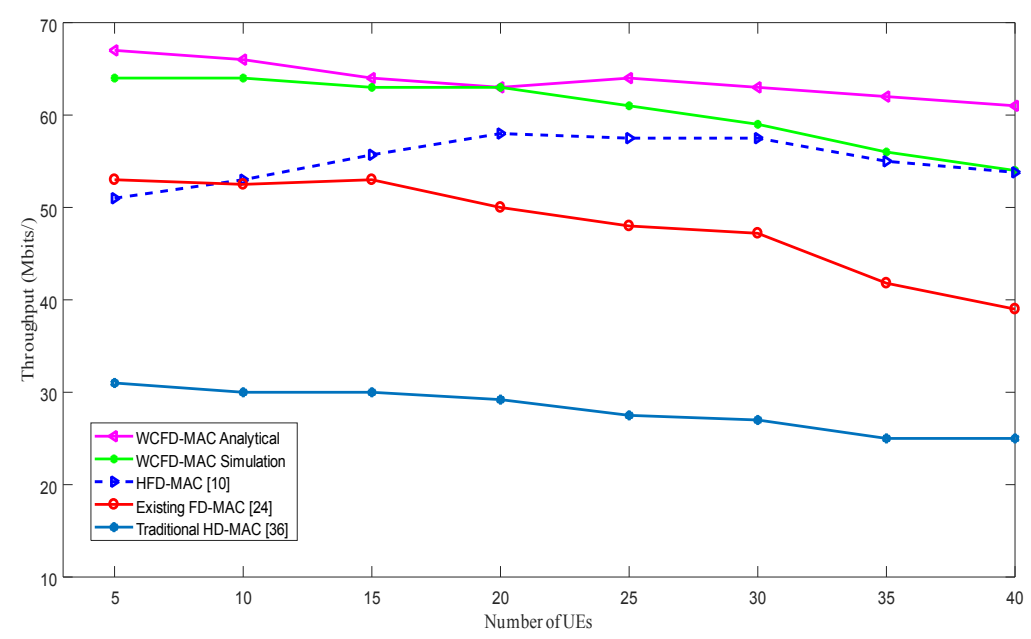

Fig. 11. Throughput with respect to the number of UEs.

1) System throughput and comparison with previous works: Fig. 11 shows the performance of the system throughput as a function of the number of UEs of our proposed MAC protocol. Fig. 11 indicates that both WCFD-MAC analytical and WCFD-MAC simulation achieve higher throughput. For our analytical throughput derivation, we used (22) where the proportion of occurrence of the FD links $\omega$ was fixed to 0.90 , while for the simulation throughput derivation, the proportion of occurrence of the FD links $\omega$ varies according to the given threshold value of angle for TAC, which was not considered in the analytical throughput calculation. The threshold angle value for TAC was fixed to $6^{\circ}$ ( 6 degrees). In other words, when the angle $\theta$ is lower than $6^{\circ}$, the corresponding ST or SR cannot be selected for the ongoing TNFD communication. Therefore, the values of the two throughputs are different, but they have similar behavior and approximatively similar values, which can justify our simulation throughput derivation approach. Also, other parameters such as $\gamma$ and $k$ were fixed to 0.3 (for a fair comparison with HFD-MAC) and 1 (perfect SI cancellation), respectively. From now, the comparison of our system throughput with that of the previous works, will only consider WCFD-MAC simulation throughput. The throughput of WCFD-MAC protocol varies around the average value of $60 \mathrm{Mbits} / \mathrm{s}$ while the throughput of HFD-MAC [10] varies around 54,29 Mbits/s, the throughput of FD-MAC [24] varies around 48,06 Mbits/s and that of HD-MAC (IEEE 80.11 standard) varies around 28,087 Mbits/s. WCFD-MAC protocol achieves a throughput gain of $213 \%$ with respect to the traditional HD-MAC protocol. Our proposed WCFD-MAC achieves higher system throughput than FD-MAC [24] since the considered work did not consider TNFD communication. For the simulation of HFD-MAC [10], the authors have fixed $30 \%$ of total nodes as hidden terminals where inter-user interference is suppressed by avoiding TNFD transmission when the two corresponding UEs are closer. This mechanism decreases the spatial reuse, thus decreasing the overall 
system throughput since TNFD will only occur when the ST and PR or when the PT and SR are out of the transmission range of each other, which will impose a high probability of HD communication than TNFD. WCFD-MAC protocol achieves higher throughput than HFD-MAC because our design simply requires a TAC to be satisfied, thus it allows TNFD communications with high probability with respect to HFD-MAC [10], thus, enhancing spectrum efficiency and special reuse. Another major problem with HFD-MAC protocol is that in a source-based TNFD communication scenario the UEs must perform a self-timer algorithm and the user whose self-timer reaches zero will become the ST. This algorithm also may decrease the system throughput since it introduces more delay, which may not satisfy 5G cellular networks delay requirement. In WCFD-MAC protocol, the self-timer algorithm is suppressed, in order to minimize communication delay and enhance the system throughput. Hence, our proposed design enhances the spectrum efficiency by allowing better special reuse than all the existing FD MAC protocols, and it can at least double the system throughput of the traditional HD system.

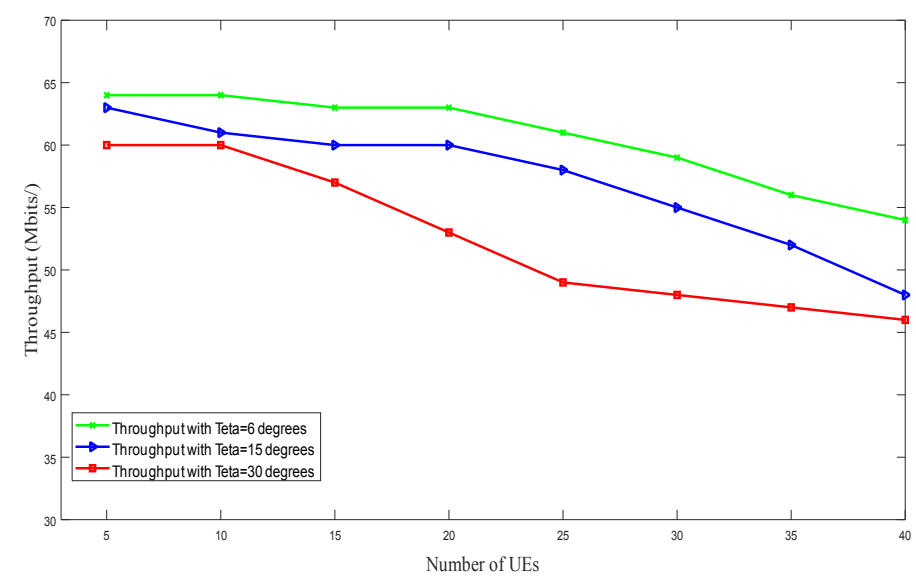

Fig. 12. Impact of threshold angle on the throughput with $\mathrm{k}=1$.

2) Impact of the threshold value of angle on the throughput: Fig. 12 shows the variation of the system throughput with the number of the UEs in the covered cell with respect to different values of the threshold angle values assuming a perfect SI cancellation. When the value of the given threshold angle increases, the system throughput decreases, as shown in Fig. 12 with $6^{\circ}, 15^{\circ}$ and $30^{\circ}$ of the threshold value of angle. Additionally, Fig. 12 indicates that when the threshold angle for $\theta$ is $30^{\circ}$ the decrease of system throughput becomes more important. This is due to the fact that the number of the UEs that cannot be selected for TNFD communication (because they cannot satisfy the established TAC) becomes high, thus imposing more HD communications, which may decrease the throughput. When the value of the threshold angle for $\theta$ is very small, for example, when this value is fixed to $6^{\circ}$, the achieved throughput at least doubles that of the traditional HD system of the IEEE 802.11 standard. Since beamforming/ directional transmission in $5 \mathrm{G}$ networks is assumed to be performed using massive MIMO and mmWave technologies, the beamwidths of the UEs and BS may be very 
small, thereby allowing small threshold value for $\theta$, which may further enhance the network throughput. Note that the beam width of the antenna at UE side may be different from that of the BS, but the angle $\theta$ is derived only using the beam width of the UEs in both, source-based TNFD and destination-based TNFD scenarios. Hence, the beam width of the BS cannot affect the system throughput of the proposed design, because TAC aims to mitigate the inter-user interferences.

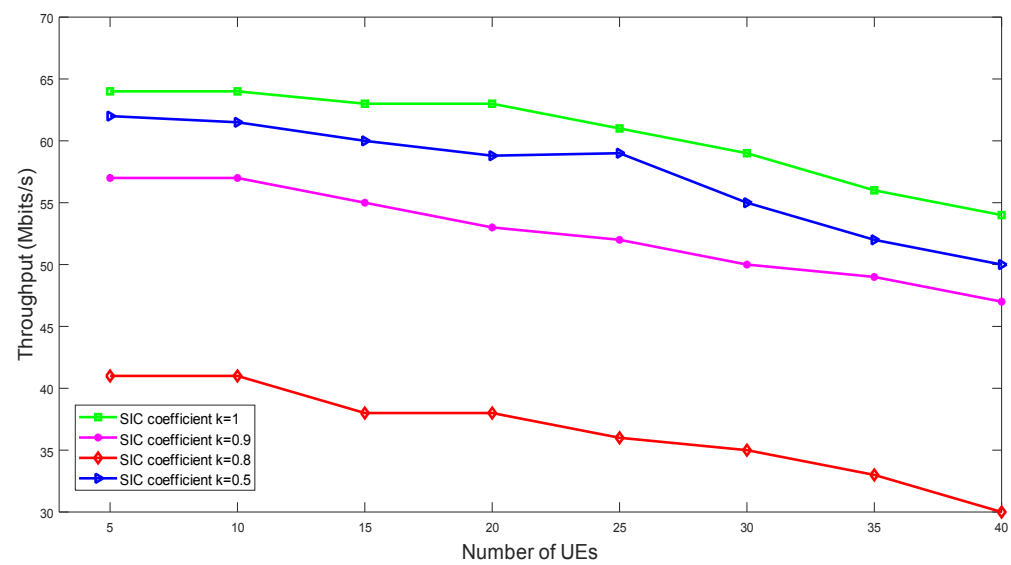

Fig. 13. Impact of SI cancellation coefficient (SIC) on the system throughput with $\theta=6^{\circ}$ ( 6 degrees).

3) Impact of the SI cancellation coefficient on the system throughput: Another factor that affects the system throughput is the value of the SI cancellation coefficient $k$. Fig. 13 shows how the system throughput decreases when the SI cancellation algorithm is not efficient at the FD devices, including the BS and UEs. Different values of the SI cancelation coefficient $k$ will have a different impact on system throughput. In Fig. 13, it is shown that when the SI coefficient $k$ is 1 or approaches 1 ( $k=0.9$ ), the achieved throughput at least doubles the throughput of the traditional HD system of the IEEE 802.11 standard. However, when $k$ is 0.5 or less than this value 0.5 , the achievable throughput is approximatively equal to or less than the throughput of the HD communication system. Better performance of SI cancellation obtained by combining passive suppression, analog and digital domain cancellations can further enhance the system throughput with a value of $k$ that reaches 1 .

4) Energy efficiency (EE): Fig. 14 shows the energy efficiency (EE) variation with respect to packet sizes for different communication schemes using WCFD-MAC. In each case, the EE was derived as

$$
E E=\frac{E_{T x}}{E_{T x}+E_{C o l}}
$$

where, $E_{C o l}$ denotes the energy consumed in collided control frame transmission. $E_{T x}$ denotes the total energy consumed in BFD, TNFD, HD or TNHD successful transmission, respectively, and it was derived using the expressions of energy consumption for FD transmission as in [43]. For further details on the calculation of these parameters, the interested reader is directed to [43] and the 
references within. For all the communication scenarios depicted in Fig. 14, the EE is enhanced when the data packet size grows. Furthermore, as depicted in Fig. 14, BFD scheme achieves high EE than TNFD and HD schemes. TNHD scheme enhances the EE of the cell boundary UE in HD communication scenario.

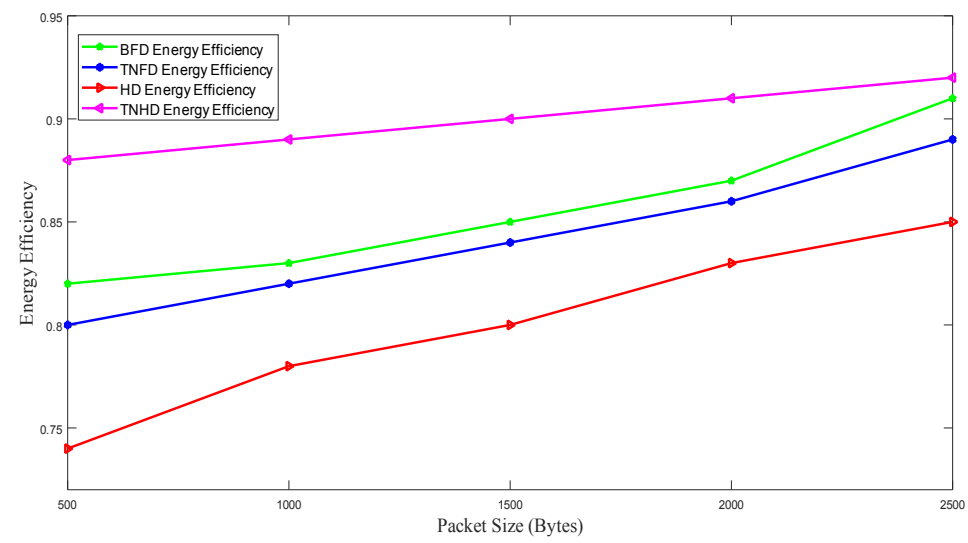

Fig. 14. Energy efficiency of different communication schemes.

5) Spectral efficiency (SE): The well-known throughput expression of Shannon is $B \log _{2}(1+S N R)$, where B is the frequency band. From the relationship between the throughput expressed in bits/s and the SE expressed in bits $/ \mathrm{s} / \mathrm{Hz}$ and assuming $\mathrm{B}=1 \mathrm{~Hz}$, the $\mathrm{SE}$ is equivalent to the throughput. Since WCFD-MAC protocol doubles the achieved throughput of the traditional HD scheme, the SE of WCFD-MAC protocol also doubles the achieved SE of the traditional HD scheme.

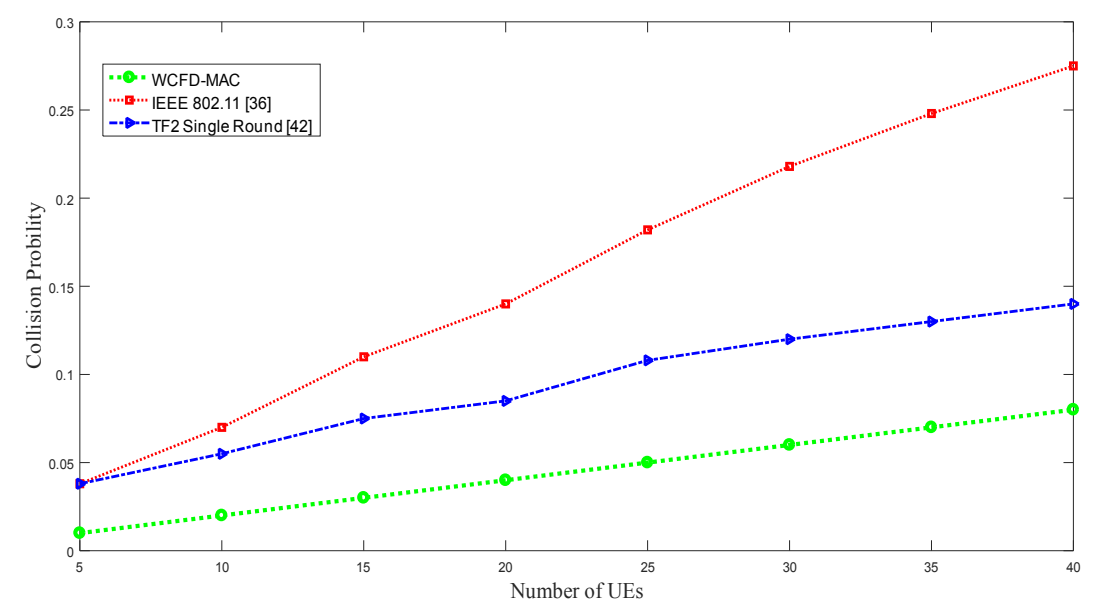

Fig. 15. Comparison of collision probabilities.

6) Collision probability: Fig. 15 shows a comparison of the collision probabilities between WCFD-MAC protocol, T2F Single Round protocol presented in [42] and IEEE 802.11 standard basic scheme [36]. For all these MAC protocols, the collision probability grows proportionally with the number of UEs in the covered small cell, because when increases the number of the contending UEs, more UEs will try to access the channel simultaneously, which will result in a collision increase. As 
shown in Fig. 14, WCFD-MAC achieves the lowest collision probability even when increases the number of UEs in the covered cell. This high performance is due to the RTSD/CTSD mechanism which is omnidirectionally transmitted for the channel reservation in order to prevent collisions. Hence, our proposed WCFD-MAC protocol achieves high performance in terms of collision probability.

\section{CONCLUSION AND FUTURE WORKS}

In this paper, we designed WCFD-MAC protocol for co-existing WLANs in 5G cellular networks considering all possible communications schemes in an FD covered small cell including BFD, TNFD and HD. To mitigate the inter-user interferences in TNFD communications, we proposed an optimal TAC based on the width of the beams of the antennas, AOA, and mathematical cosine and sine rules. The proposed TAC allows two neighbor UEs to simultaneously participate in TNFD communication without interfering with each other. This increases the overall system throughput of the covered cell. Our MAC protocol includes a new HD (TNHD) communication scheme that aims to enhance the throughput of the small cell boundary UE and enhance energy efficiency by reducing the energy consumption at both, the BS and UE. The achieved throughput of WCFD-MAC protocol was compared to the previous works, namely HFD-MAC, FD-MAC, and traditional HD IEEE 802.11 standard. It has been shown that WCFD-MAC protocol performs higher throughput than these works and it can double the throughput of the HD IEEE 802.11 standard when the given threshold value for $\mathrm{TAC}$ is small or equal to $15^{\circ}$ assuming perfect SI cancellation. However, when the SI cancellation coefficient reaches 0.5 WCFD-MAC achieve approximatively the same throughput of the IEEE 802.11 standard with the threshold angle value for TAC fixed to $6^{\circ}$. Due to significant performance improvements and the fact that it considers some important features of $5 \mathrm{G}$ networks for application in cellular scenarios the proposed protocol can be a solution for the co-existing (integrated and nonintegrated) WLANs in future $5 \mathrm{G}$ mobile networks.

When estimating the angle $\theta$ at the BS side in order to verify the TAC, we assumed cooperation between the BS of the covered small cell and other BSs in its vicinity, since the 5G network densification strategy requires coordination/cooperation between different BSs. In current cellular networks, there is coordination/cooperation among the BSs, however, there is a need to develop a coordination/cooperation mechanism between the cellular BSs and WLAN BSs for the 5G heterogeneous networks. When the cooperation mechanism is performed among BSs (including the cellular BSs and WLAN BSs), the estimation of the angle $\theta$ has lower complexity. In our future work, we will focus on implementing a more efficient power saving algorithm by developing a user scheduling approach that takes into account power allocation and coordination/cooperation among different BSs in a dense deployment scenario. Moreover, we have only considered symmetric traffic 
between the uplink and downlink. An interesting research topic will be how to improve WCFD-MAC protocol by taking into account asymmetric traffic conditions.

\section{ACKNOWLEGMENT}

The authors acknowledge the financial support of Programme de Formation des Formateurs (PFF) of the government of Mali, and materials support of Universidad Tecnológica de La Habana of Cuba.

\section{REFERENCES}

[1] B. Ismaiel, M. Abolhasan, D. Smith, W. Ni, and D. Franklin, "Scalable MAC protocol for D2D communication for future 5G networks," in 2017 14th IEEE Annual Consumer Communications \& Networking Conference (CCNC), USA, 2017, pp. 542-547.

[2] E. Dahlman, S. Parkvall, and J. Skold, 5G NR: The next generation wireless access technology. Academic Press, 2018.

[3] I. Ahmed et al., "A survey on hybrid beamforming techniques in 5G: Architecture and system model perspectives," IEEE Commun. Surv. Tutor., vol. 20, no. 4, pp. 3060-3097, 2018.

[4] M. S. Islam, T. Jessy, M. S. Hassan, K. Mondal, and T. Rahman, "Suitable beamforming technique for 5G wireless communications," in 2016 International Conference on Computing, Communication and Automation (ICCCA), India, 2016, pp. 1554-1559.

[5] Y.-B. Ko, J.-M. Choi, and N. H. Vaidya, "MAC protocols using directional antennas in IEEE 802.11 based ad hoc networks," Wirel. Commun. Mob. Comput., vol. 8, no. 6, pp. 783-795, 2008.

[6] I. Selinis, K. Katsaros, M. Allayioti, S. Vahid, and R. Tafazolli, "The race to 5G era; LTE and Wi-Fi," IEEE Access, vol. 6, pp. 56598-56636, 2018.

[7] M. Höyhtyä, O. Apilo, and M. Lasanen, "Review of latest advances in 3GPP standardization: D2D communication in 5G systems and its energy consumption models," Future Internet, vol. 10, no. 1, p. 3, 2018.

[8] H. Huang, W. Xiang, Y. Tao, B. Liu, and M. Hu, "Relay-Assisted D2D Transmission for Mobile Health Applications," Sensors, vol. 18, no. 12, p. 4417, 2018.

[9] Y. Cai, Y. Ni, J. Zhang, S. Zhao, and H. Zhu, "Energy efficiency and spectrum efficiency in underlay device-to-device communications enabled cellular networks," China Commun., vol. 16, no. 4, pp. 16-34, 2019.

[10] M. A. Alim, M. Kobayashi, S. Saruwatari, and T. Watanabe, "In-band full-duplex medium access control design for heterogeneous wireless LAN," EURASIP J. Wirel. Commun. Netw., vol. 2017, no. 1, p. 83, 2017.

[11] A. Aijaz and P. Kulkarni, "Protocol design for enabling full-duplex operation in next-generation IEEE 802.11 WLANs," IEEE Syst. J., no. 99, pp. 1-12, 2017.

[12] S. Diakite and F. M. Rizo, "Self-Interference Cancellation in MIMO Full-Duplex Transceivers," Rev. Cientifica Ing. Electrónica Automática Comun., vol. 40, no. 1, pp. 59-70, 2019.

[13] B. van Liempd et al., "RF self-interference cancellation for full-duplex," in 2014 9th International Conference on Cognitive Radio Oriented Wireless Networks and Communications (CROWNCOM), Finland, 2014, pp. 526-531.

[14] W. Bi, X. Su, L. Xiao, and S. Zhou, "Superposition coding based inter-user interference cancellation in full duplex cellular system," in 2016 IEEE wireless communications and networking conference, Qatar, 2016, pp. 1-6.

[15] J. Qiao, X. S. Shen, J. W. Mark, Q. Shen, Y. He, and L. Lei, "Enabling device-to-device communications in millimeterwave 5G cellular networks," IEEE Commun. Mag., vol. 53, no. 1, pp. 209-215, 2015.

[16] D. Della Penda, L. Fu, and M. Johansson, "Energy efficient D2D communications in dynamic TDD systems," IEEE Trans. Commun., vol. 65, no. 3, pp. 1260-1273, 2016.

[17] R. Q. Hu and Y. Qian, "An energy efficient and spectrum efficient wireless heterogeneous network framework for 5G systems," IEEE Commun. Mag., vol. 52, no. 5, pp. 94-101, 2014.

[18] J. Li, "Resource allocation in 5G WLAN/cellular integrated network with partially overlapped channels," PhD Thesis, 2017.

[19] K. Sakaguchi et al., "Millimeter-wave wireless LAN and its extension toward 5G heterogeneous networks," IEICE Trans. Commun., vol. 98, no. 10, pp. 1932-1948, 2015.

[20] R. Ali, N. Shahin, R. Bajracharya, B.-S. Kim, and S. W. Kim, "A self-scrutinized backoff mechanism for IEEE 802.11 ax in 5G unlicensed networks," Sustainability, vol. 10, no. 4, p. 1201, 2018.

[21] R. Alkhansa, H. Artail, and D. M. Gutierrez-Estevez, "LTE-WiFi carrier aggregation for future 5G systems: A feasibility study and research challenges," Procedia Comput. Sci., vol. 34, pp. 133-140, 2014.

[22] S. Okasaka et al., "Proof-of-concept of a millimeter-wave integrated heterogeneous network for 5G cellular," Sensors, vol. 16, no. 9, p. 1362, 2016.

[23] R. Ali, N. Shahin, R. Bajracharya, B.-S. Kim, and S. W. Kim, "A self-scrutinized backoff mechanism for IEEE 802.11 ax in 5G unlicensed networks," Sustainability, vol. 10, no. 4, p. 1201, 2018.

[24] X. Zhang, W. Cheng, and H. Zhang, "Full-duplex transmission in PHY and MAC layers for 5G mobile wireless networks," IEEE Wirel. Commun., vol. 22, no. 5, pp. 112-121, 2015. 
[25] L. M. de Guimarães and J. L. Bordim, "FDDS-MAC: Enhancing spectrum usage on full-duplex communications in 5G mobile wireless networks," in 2018 IEEE Symposium on Computers and Communications (ISCC), Brazil, 2018, pp. $00268-00273$.

[26] D. Marlali and O. Gurbuz, "Design and performance analysis of a full-duplex MAC protocol for wireless local area networks," Ad Hoc Netw., vol. 67, pp. 53-67, 2017.

[27] M. Amin, M. Hossain, and M. Atiquzzaman, "In-Band Full Duplex Wireless LANs: Medium Access Control Protocols, Design Issues and Their Challenges," Information, vol. 11, no. 4, p. 216, 2020.

[28] N. Singh, D. Gunawardena, A. Proutiere, B. Radunovi, H. V. Balan, and P. Key, "Efficient and fair MAC for wireless networks with self-interference cancellation," in 2011 International Symposium of Modeling and Optimization of Mobile, Ad Hoc, and Wireless Networks, USA, 2011, pp. 94-101.

[29] H. Wang, S. Tan, and J. Zheng, "FD-MAC: a flow-driven MAC protocol for mobile ad hoc networks," in GLOBECOM 2009-2009 IEEE Global Telecommunications Conference, USA, 2009, pp. 1-5.

[30] J. Seddar, H. Khalifé, W. Al Safwi, and V. Conan, "A full duplex MAC protocol for wireless networks," in 2015 international wireless communications and mobile computing conference (IWCMC), Croatia, 2015, pp. 244-249.

[31] J. Y. Kim, O. Mashayekhi, H. Qu, M. Kazandjieva, and P. Levis, "Janus: A novel MAC protocol for full duplex radio," CSTR, vol. 2, no. 7, p. 23, 2013.

[32] W. Cheng, X. Zhang, and H. Zhang, "RTS/FCTS mechanism based full-duplex MAC protocol for wireless networks," in 2013 IEEE Global Communications Conference (GLOBECOM), USA, 2013, pp. 5017-5022.

[33] J. Jang et al., "Smart small cell with hybrid beamforming for 5G: Theoretical feasibility and prototype results," IEEE Wirel. Commun., vol. 23, no. 6, pp. 124-131, 2016.

[34] A. B. Pandya and B. D. Parmar, "The Impact of Directional Antennas on MAC Layer Protocol for Ad-Hoc Networks," Int. J. of Computer sci. and Tech., vol. 3, no. 1, pp. 348-352, 2012.

[35] O. W. Abdulwahhab, "Mobile Position Estimation based on Three Angles of Arrival using an Interpolative Neural Network," Int. J. Comput. Appl., vol. 100, no. 7, 2014.

[36] I. 80211 W. Group, "Part 11: Wireless LAN Medium Access Control (MAC) and Physical Layer (PHY) specifications: Higher-speed physical layer in the 5GHz band," IEEE Std 80211, 1999. [Online]. Available: IEEE Xplore, https://ieeexplore.ieee.org. [Accessed Dec. 12, 2019].

[37] S. Goyal, P. Liu, O. Gurbuz, E. Erkip, and S. Panwar, "A distributed MAC protocol for full duplex radio," in 2013 Asilomar Conference on Signals, Systems and Computers, CA, USA, 2013, pp. 788-792.

[38] R. Roy and T. Kailath, "ESPRIT-estimation of signal parameters via rotational invariance techniques," IEEE Trans. Acoust. Speech Signal Process., vol. 37, no. 7, pp. 984-995, 1989.

[39] N. A. Baig and M. B. Malik, "Comparison of direction of arrival (DOA) estimation techniques for closely spaced targets," Int. J. Future Comput. Commun., vol. 2, no. 6, p. 654, 2013.

[40] Bianchi Giuseppe, "Performance analysis of the IEEE 802.11 distributed coordination function," IEEE J. Sel. Areas Commun., vol. 18, no. 3, pp. 535-547, 2000.

[41] L. de M. Guimarães and J. L. Bordim, "A Full-duplex MAC tailored for 5G Wireless Networks," Wirel. Commun. Mob. Comput., vol. 2018, 2018.

[42] S. Sen, R. R. Choudhury, and S. Nelakuditi, "Listen (on the frequency domain) before you talk," in Proceedings of the 9th ACM SIGCOMM Workshop on Hot Topics in Networks, 2010, p. 16.

[43] M. O. Al-Kadri, A. Aijaz, and A. Nallanathan, "An energy-efficient full-duplex MAC protocol for distributed wireless networks,” IEEE Wirel. Commun. Lett., vol. 5, no. 1, pp. 44-47, 2015. 